\title{
Space Weather
}

\section{REVIEW ARTICLE}

10.1002/2016SW001501

\section{Special Section: \\ NASA's Living With a Star: Geomagnetically Induced Currents}

\section{Key Points:}

- We provide a broad overview of the status of the GIC field

- We utilize the Applications Readiness Levels (ARL) concept to quantify the maturity of our GIC-related modeling and applications

- This paper is the high-level report of the NASA Living With a Star GIC Working Group findings

Correspondence to:

A. Pulkkinen,

antti.a.pulkkinen@nasa.gov

Citation:

Pulkkinen, A., et al. (2017), Geomagnetically induced currents: Science, engineering, and applications readiness, Space Weather, 15, 828-856, doi:10.1002/2016SW001501.

Received 14 AUG 2016 Accepted 26 JAN 2017 Accepted article online 30 JAN 2017 Published online 6 JUL 2017

\section{Geomagnetically induced currents: Science, engineering, and applications readiness}

\author{
Pulkkinen A. $^{1}$ (D) E. Bernabeu², A. Thomson ${ }^{3}$, A. Viljanen ${ }^{4}$, R. Pirjola ${ }^{4,5}$ (D) D. Boteler ${ }^{5}$, J. Eichner ${ }^{6}$, \\ P. J. Cilliers' ${ }^{7}$, D. Welling ${ }^{8}$ (D) N. P. Savani ${ }^{\text {iD, R. S. Weigel }}{ }^{10}$ (D) J. J. Love $^{11}$ (D, C. Balch ${ }^{12}$ (D), \\ C. M. Ngwira ${ }^{1,13}$ (D) G. Crowley $^{14}$ (D, A. Schultz ${ }^{15}$, R. Kataoka ${ }^{16}$ (D) B. Anderson ${ }^{17}$ (D), D. Fugate ${ }^{18}$, \\ J. J. Simpson ${ }^{19}$ iD, and M. MacAlester ${ }^{20}$
}

${ }^{1}$ NASA Goddard Space Flight Center, Greenbelt, Maryland, USA, ${ }^{2}$ PJM, Valley Forge, Pennsylvania, USA, ${ }^{3}$ British Geological Survey, Nottingham, UK, ${ }^{4}$ Finnish Meteorological Institute, Helsinki, Finland, ${ }^{5}$ Natural Resources Canada, Ottawa, Ontario, Canada, ${ }^{6}$ Munich-Re, Munich, Germany, ${ }^{7}$ South African National Space Agency, Pretoria, South Africa, ${ }^{8}$ Department of Atmospheric, Oceanic, and Space Sciences, University of Michigan, Ann Arbor, Michigan, USA, ${ }^{9}$ Goddard Planetary Heliophysics Institute, University of Maryland, Baltimore County, Baltimore, Maryland, USA, ${ }^{10}$ Department of Physics and Astronomy, George Mason University, Fairfax, Virginia, USA, ${ }^{11}$ U.S. Geological Survey, Golden, Colorado, USA, ${ }^{12}$ NOAA Space Weather Prediction Center, Boulder, Colorado, USA, ${ }^{13}$ Institute for Astrophysics and Computational Sciences, Catholic University of America, Washington, District of Columbia, USA, ${ }^{14}$ Atmospheric and Space Technology Research Associates, LLC, Boulder, Colorado, USA, ${ }^{15}$ Institute for Energy Resources and Resilience, Oregon State University, Corvallis, Oregon, USA, ${ }^{16}$ National Institute of Polar Research, Tokyo, Japan, ${ }^{17}$ The Johns Hopkins University Applied Physics Laboratory, Laurel, Maryland, USA, ${ }^{18}$ Electric Research and Management, Inc., Cabot, Pennsylvania, USA, ${ }^{19}$ Electrical and Computer Engineering Department, University of Utah, Salt Lake City, Utah, USA, ${ }^{20}$ Federal Emergency Management Agency, Washington, District of Columbia, USA

Abstract This paper is the primary deliverable of the very first NASA Living With a Star Institute Working Group, Geomagnetically Induced Currents (GIC) Working Group. The paper provides a broad overview of the current status and future challenges pertaining to the science, engineering, and applications of the GIC problem. Science is understood here as the basic space and Earth sciences research that allows improved understanding and physics-based modeling of the physical processes behind GIC. Engineering, in turn, is understood here as the "impact" aspect of GIC. Applications are understood as the models, tools, and activities that can provide actionable information to entities such as power systems operators for mitigating the effects of GIC and government agencies for managing any potential consequences from GIC impact to critical infrastructure. Applications can be considered the ultimate goal of our GIC work. In assessing the status of the field, we quantify the readiness of various applications in the mitigation context. We use the Applications Readiness Level (ARL) concept to carry out the quantification.

\section{Introduction}

Geomagnetic disturbances (GMD) cause geomagnetically induced currents (GIC) to flow in long engineered conductor systems such as power grids, pipelines, and railway systems. GIC have become one of the main space weather concerns, and the potential for widespread problems in operating high-voltage power transmission systems during major geomagnetic storms has prompted increasing international policy, science, industry, and public interest in the problem. In the U.S., the latest high-level attention on GIC and power grids is centered around regulatory action initiated by the Federal Energy Regulatory Commission and GIC-related elements of the National Space Weather Strategy and National Space Weather Action Plan [United States of America Federal Energy Regulatory Commission, 2013; National Science and Technology Council, 2015a; National Science and Technology Council, 2015b]. In the UK, GIC are part of the space weather element in the National Risk Registry [Cabinet Office, 2015]. In addition, the power transmission industry is quickly elevating awareness to address the GIC issue, acknowledging that the problem pertains to middle and low latitudes as well as high latitudes [e.g., Gaunt and Coetzee, 2007; Liu et al., 2009; Torta et al., 2012; Carter et al., 2015]. Consequently, power system operators in nations such as the US, UK, Canada, Finland, Norway, Sweden, China, Japan, Brazil, Namibia, South Africa, and Australia have launched GIC measurement and hazards assessment campaigns to understand and mitigate the possible GIC impact on their systems. The field of GIC has evolved over the past several years from a somewhat separate field of space science research into a full systems science addressing not 
only the solar-terrestrial research but also the engineering and operational hazard mitigation dimensions of the problem.

In recognition of the rapidly growing interest in the topic, this paper presents the findings of the very first NASA Living With a Star (LWS) Institute Working Group that specifically targeted the GIC issue. NASA launched the new LWS Institutes program element in 2014. The concept is built around small working group style meetings that focus on well-defined problems that demand intense, direct interactions between colleagues in neighboring disciplines. This facilitated the development of a deeper understanding of the variety of processes that link solar activity to Earth's environment. The LWS Institute GIC Working Group, led by A. Pulkkinen (NASA Goddard Space Flight Center) and co-led by E. Bernabeu (PJM) and A. Thomson (British Geological Survey), was selected competitively as the pilot activity for the new LWS element. The coauthors of this paper are the core members of the LWS Institute GIC Working Group tasked to (1) identify, advance, and address the open scientific and engineering questions pertaining to GIC, (2) advance predictive modeling of GIC, and (3) advocate and act as a catalyst to identify resources for addressing the multidisciplinary topic of GIC. The group had two 5 day in-person workshops in Colorado and several half-day videoconferences to develop the group materials and facilitate new collaborative GIC research activities.

In this paper, we target the task (1) of the LWS Institute GIC Working Group. More specifically, this paper captures the current status and future challenges pertaining to science, engineering, and applications of the GIC problem (for similar work, see also Thomson et al. [2010] and Love et al. [2014]). Science is understood here as the basic space and Earth sciences research that allow improved understanding and physics-based modeling of the physical processes behind GIC. Engineering is understood as the "impact" aspect of GIC. The impact includes any physical effects that GIC may have on the performance of technological infrastructure. While we acknowledge that the loss of electricity can lead to major follow-on consequences that can potentially be disruptive to society, we will not discuss impacts beyond engineering considerations in this paper. Applications is understood as the models, tools, and activities that can provide actionable information to entities such as power systems operators for mitigating the effects of GIC and government for managing any potential consequences from GIC impact to critical infrastructure. In this sense, applications can be considered as the ultimate goal of our GIC work. In assessing the status of the field, we quantify the readiness of various applications in the GIC effects mitigation context.

The structure of the paper is as follows. In section 2, we give further motivation for GIC work from the perspective of the operational organizations represented in the GIC Working Group. Section 2 also outlines what type of information is needed from the operational organizations' standpoint to address the issue. In section 3, we provide a comprehensive outline of the space weather chain and corresponding links from the solar atmosphere down to the upper mantle of the Earth. While the topics in section 3 cover a wide variety of space weather processes, the discussion is carried out primarily in the GIC context. Section 3 also provides the connection to the engineering dimension of the topic and discusses some of the key open questions pertaining to GIC. Further, section 3 gives an initial quantification of our current applications readiness to address the GIC issue. Building on the last two parts of section 3, section 4 provides further discussion about GIC impacts. Finally, in section 5 we provide general discussion about the findings of the team. Appendix A outlines further details about the open scientific questions identified by the team and "project templates" that were developed to address those questions.

We note that GIC impact not only electric power transmission systems but also oil and gas pipelines, railway systems, and any other extended ground-based conductor systems having length scales of the order of $\sim 1$ km or more (e.g., Boteler et al. [1998]; see also Knipp [2015], for commentary and an extensive collection of GIC research papers; for a review of space weather science in general, see Schrijver et al. [2015]). While most of the discussion in this paper pertains generally to all of GIC, in the engineering and impacts discussions in sections 3.7, 3.8, and 4, we focus only on high-voltage power transmission systems. The focus on power transmission systems was motivated by the desire to confine the group's work and the fact that most of the GIC concerns at this time pertain to impacts on power grids.

\section{Why Do We Care and What Is Needed?}

While GIC has recently received elevated attention from the research community, industry, and government, it is helpful to revisit the question "why do we care?" Since the LWS Institute GIC Working Group included 
representatives from different types of end user and operational organizations, one of the first assignments for the group was to specify the reasons why the corresponding organizations think GIC is an important problem and what is needed to address the problem. We asked the group's electric power transmission industry, National Oceanic and Atmospheric Administration (NOAA), U.S. Geological Survey (USGS), Federal Emergency Management Agency (FEMA), and insurance industry representatives to express their views on the topic, which are provided below.

\subsection{Power Transmission Industry View (by E. Bernabeu, PJM)}

GIC are the manifestation of space weather driven by solar activity. Disturbances on Earth's geomagnetic field induce a geoelectric field at the Earth's surface, which drives GIC. Since frequencies smaller than $1 \mathrm{~Hz}$ dominate the power spectrum of the geoelectric field, GIC behaves like a DC current when compared to the 50 or $60 \mathrm{~Hz}$ AC power systems (see section 3.6 for further explanation). These quasi-DC (as also called "zero sequence" in the engineering terminology) currents flow through transmission lines and enter/exit the power grid through grounded transformer neutrals.

The flow of GIC can drive power transformers into half-cycle saturation, increasing the reactive power consumed by the transformer, injecting even and odd harmonics into the system, and potentially generating hot spots in the windings and/or structural components (see section 3.7 for further explanation). Combined, these effects may result in equipment loss of life, equipment damage, and/or a system-wide disturbance; the most famous impact of a geomagnetic disturbance is the Hydro-Quebec blackout in March 1989 [e.g., Bolduc et al., 2000; Bolduc, 2002].

In order to assess the risk to the electric power system, we first need a meaningful characterization of GMD events. The scientific community has numerous parameters to describe geomagnetic disturbances: such as $D s t, K p, A p$, and $\mathrm{d} B / \mathrm{d} t$. However, from the engineering point of view, the spatial-temporal characteristics of the horizontal geoelectric field provide the ideal description of a GMD event.

The distinctive characteristic of GMDs, when compared to other Earth weather phenomena, is its wide-area nature. A geomagnetic disturbance can engulf the entire North American continent. However, it is important to note that it is not the instantaneous magnetic field footprint that dictates GIC but the fluctuations in the field. And the fluctuations can be very complex and localized. Consequently, while wide areas can be exposed to the storm footprint, the very highest levels of GIC can be regional or local in nature. Also, the severity of the storm and its associated risk is a strong function of geomagnetic latitude and ground conductivity structure. Consequently, the geoelectric field (uniform or nonuniform) needs to be described at spatial scales relevant to the bulk power system (hundreds of kilometers). The spectral signature of the geoelectric field is also a key input parameter to assess the thermal response of power transformers.

GMD benchmark scenarios (for example, a 1-in-100 year event) that properly describe the spatial-temporal characteristics of the geoelectric field are the first step in the risk analysis process. Aided by these scenarios, power system engineers can identify critical locations in the system, plan and harden the system to improve resiliency, and develop corrective actions to mitigate the risk.

A resilient power system must be able to reduce the magnitude and/or duration of any potential disruptive event. The power grid should not only be robust against GMD events (planning and hardening) but also be able to anticipate, adapt, and recover. Currently, state-of-the-art forecasts issued by NOAA are used to posture the system (long 1-2 day lead time forecast) and to provide situational awareness and adapt operating conditions during the storm (short 15-30 min lead time forecast). Improving forecasting tools to specify regional (as opposed to global) geoelectric fields will allow operators to manage risk in a cost-effective manner.

Assessing the risk on the electric grid is a complex and challenging endeavor. The risk assessment must consider the interaction between multiple interdependent models: transformer thermal response, transformer electromagnetic models, voltage stability, system-wide harmonic propagation, protection, and control. The wide-area nature of GMD further complicates the analysis, making interregional coordination an essential aspect of risk mitigation.

Significant efforts have been made to incorporate the impacts of GIC into traditional power system studies. Despite the rapid improvement of power system analysis tools, it is well recognized that gaps still exist both from the scientific and engineering points of views. These tools will evolve and improve as our knowledge of GMD and understanding of its impacts matures. Model validation will play an essential role in this process, and it will require a strong interaction between scientists, engineers, manufacturers, and government agencies. 


\subsection{NOAA Space Weather Prediction Center View (by C. Balch, NOAA SWPC)}

NOAA's Space Weather Prediction Center's (SWPC) mission is to provide space weather alerts, warnings and forecasts to the nation and the world. A key impact area concerns GIC in the power grid. In addition to providing regular operational space weather information, SWPC has an active interest in new findings and understanding for this application, with an intention of modernizing space weather products and forecasts to incorporate sufficiently well-developed concepts to meet end user needs. Through interactions with the LWS Institute GIC Working Group, SWPC has gained a better appreciation of user requirements for the specification and prediction of the geoelectric field, and insights into the state-of-the art regarding current and future possibilities for meeting these requirements. There are a number of areas which present a significant challenge to current capabilities. One of the leading sources of uncertainty is the predictions of the strength and orientation of the interplanetary magnetic field of coronal mass ejections that may impact Earth. Another key advance is the ongoing transition of physics-based and empirical models to predict the response of the magnetosphere and ionosphere to observations taken upstream from the Earth at the Lagrange 1 (L1) position by the ACE and DSCOVR missions. These models will enable the first steps into the production of region-specific forecasts for geomagnetic activity that are needed by electrical power system operators. Encouraging as this is, however, we note that recent observations of the large-scale fieldaligned current structures captured in the Active Magnetosphere and Polar Electrodynamics Response Experiment (AMPERE) data [Anderson et al., 2000] indicate that there are still challenges ahead for these kinds of models to predict the details of the activity that is actually observed. Finally, key advances in observations of the ground electromagnetic response characteristics resulting from the EarthScope project across parts of the United States promise to significantly improve the nowcast specification of space weather in terms of the geoelectric field, which is ultimately what is required for this application area.

\subsection{USGS View (by J. Love, USGS)}

Ground-based data are of fundamental importance for long-term forecasting, real-time monitoring, and prospective evaluation of space weather conditions and induction-related hazards. The Geomagnetism Program of the U.S. Geological Survey (USGS) is collaborating with other space weather agencies to use magnetic observatory data in conjunction with magnetotelluric measurements of Earth surface impedance to estimate geoelectric fields for either general hazard assessment [Love et al., 2016a] or space weather operations [Kelbert et al., 2017]. The operation of magnetic observatories is a basic mission of the USGS, and data from the observatories have been collected for many decades, and they are available in near real time for monitoring space weather conditions [Love and Finn, 2011]. Magnetotelluric surveys are normally undertaken to estimate the electrical conductivity structure of the Earth's crust and mantle. In the U.S., the National Science Foundation has supported, through its EarthScope Program, a large-scale magnetotelluric survey of the United States [Schultz, 2010]. A fringe benefit of the EarthScope magnetotelluric impedance measurements, not widely anticipated when the program was initiated, is their utility for estimating geoelectric fields.

\subsection{FEMA View (by M. MacAlester, FEMA)}

Emergency managers and elected officials have a critical stake in space weather analytics to manage the potential consequences of a damaging event and to inform planning. An extreme space weather event such as the Carrington Event of 1859 is a hazard we have not faced in modern times. The impact on critical infrastructure systems - particularly electric power-is not well understood, though progress is being made. Every other critical infrastructure sector (i.e., communications, water, healthcare, and financial) depends on electric power. Preimpact planning requires that emergency managers know the following: the probability and associated confidence level that an extreme event will occur similar to current NOAA warnings related to tornadoes, hurricanes, and other extreme terrestrial weather events; whether a coronal mass ejection (CME) will be geoeffective (and if this can be known before it reaches L1); and the arrival time, intensity, duration, and geographic impact of the CME at Earth. NOAA SWPC would collate and provide this information to emergency managers. Immediate response and recovery planning requires information from electric utilities on potential impacts to power within their service areas and on estimates for power restoration at regionaland community-level resolutions, if possible. Emergency managers and elected officials must provide to the public authoritative, reliable, timely, and actionable information in anticipation of and in response to a damaging event. They also need to direct limited resources to maximize life-saving and life-sustaining efforts and to speed up recovery. Just as with hurricanes and tornadoes, hours and even minutes of lead time will matter. 
Finally, officials need the output of simulated geomagnetic storm scenarios to inform planning for preparedness, response, recovery, and mitigation.

\subsection{Insurance Industry View (by J. Eichner, Munich-Re)}

A central term in the insurance sector is "holistic risk" which is defined as a three-parameter function consisting of (1) the hazard (i.e., the probability of occurrence of a hazardous event at a given location), (2) the exposure (i.e., the values prone to the hazardous forces at the location), and (3) the vulnerability (i.e., the susceptibility of the exposure to the hazardous forces). The holistic risk can only be understood and managed if all three factors are known to the greatest possible extent.

Insurance cannot help to avoid impacts from severe or catastrophic events, but it can lessen the impact's consequences by mitigating the losses and providing financial help to rebuild and restart. This role puts insurance at the end of the functional economic chain. With a long history of experience in managing classical risks (stemming from hazardous events such as strong earthquakes, severe storms, and large floods) and challenges from emerging risks and risks of change, insurance has become effectively an early-warning system to the economy and to some extent to society and politics. Various statistical and scenario-based methods are applied in the insurance industry to estimate the impact dimension of so-called accumulation risk scenarios. Such scenarios describe events that cause losses in manifold ways and carry the highest loss potentials due to the complexity of the propagation of losses in interrelated socioeconomic sectors.

Solar storms and geomagnetic storms are not part of the classical business portfolio of the insurance industry. However, the topic gained momentum when a prominent impact scenario of a potential solar superstorm (such as the Carrington event in 1859) found ample attention in the media. In the scenario, developed by John Kappenman [National Research Council, 2008, pp. 77-79], hundreds of high-voltage power transformers suffer damage from GIC and a widespread and long-lasting power outage could strike North America (see section 3.7 for a review of this and other assessments of the GIC risk to the power grid). The described scenario would be too big to be insurable, not because of the direct losses stemming from defective electric infrastructure but rather from tangible and intangible losses as a consequence of long-lasting and widespread power outage with possible business interruption and even riots. But even a scaled-down and therefore more probable scenario still carries the potential of a substantial macroeconomic impact that is beyond insurance and, hence, of societal-political dimension.

Organized through the insurance industry's Geneva Association, in 2011 a working group on space weather ground effects was established with the goal to learn about the risks from extreme solar storms, and to answer the central question: is a Kappenman-type scenario a realistic potential outcome of a 100, 200, 500, or even 1000 year extreme solar storm event? Such a question addresses both the hazard probability as well as the vulnerability of the prevailing high-voltage power grid technology. Translated to GIC, one needs to study the driving parameters which, besides the influence of geological and geographical amplifications, modulate strong geoelectric fields that can occur locally or regionally. One also needs to identity if the geoelectric field enhancements come with a short duration and high magnitude or a long duration and low magnitude. Just these three parameters (magnitude, duration and geographic coverage) allow creating a virtually infinite amount of, e.g., "100 year geoelectric field" scenarios. Which ones are the ones that create the strongest GIC? Pertaining to vulnerability, one needs to study the susceptibility of the existing power grid components (such as high-voltage power transformers) to these extreme GIC scenarios. And depending on the degree of vulnerability: what can be done to reduce it?

Additional motivation for the research on extreme solar storm impacts is coming from the European corporate law. On 1 January 2016, a new European Directive on insurance regulation called "Solvency II" became effective. The directive requires every European insurance company to prove that they are financially prepared against any knowable loss event with a probability of occurrence of 1-in-200years. Realistic and reliable scenarios (including extreme space weather) will help the insurance industry to prepare for the Solvency II requirements.

\subsection{Summary of the Views}

As can be seen above, the five views have interesting distinct flavors and diversity of perspectives to the GIC problem. However, despite the distinct flavors, the five views also have substantial parallels. It is clear that the geoelectric field is the key target for scientific investigations, and improved understanding and specification 
of the field over different spatial and temporal scales is needed. There is also a need for improved understanding of the geoelectric field extremes and storm scenarios. Improved real-time specification and forecast capacity are also common themes in all subsections above and will require addressing the entire "Sun to mud" space weather chain ending in GIC. Motivated and guided by these perspectives, we tackle the Sun to mud chain including specification, modeling, and forecasting of GIC in the section below.

\section{Space Weather Chain From the Sun to Mud (and Below)}

GIC poses a special challenge in the space weather context. From the science standpoint, the phenomenon can be considered as the end link of the space weather chain extending from the Sun to the surface of the Earth and below. The GIC signal carries information about the entire chain, and for a complete understanding of the phenomenon, one needs to acquire a complete understanding of the processes operating within individual physical domains and interactions between the domains. The major progress over the past decade in understanding solar, heliospheric, magnetospheric, ionospheric, thermospheric, and solid Earth domains, and especially coupling between them, has led to a quickly maturing understanding of the key processes driving GIC.

One of the major advancements in the field has been the enhanced communications between the science and power engineering communities. These communications have helped to establish the geoelectric field, which is the physical driver of GIC, as the key interface between the disciplines. The geoelectric field requires knowledge about space physical and geophysical processes and can be used to compute GIC and the corresponding power transmission system response. In this sense, the "division of work" is now clear: science activities need to characterize the geoelectric field, which is the input for further engineering analyses. Further, since the end users now have the appropriate tools and know how to use the geoelectric field in their engineering assessments, this interface allows the engineering community to pass questions and requests back to the science community and thus facilitate the two-way exchange of actionable information.

The goal of this section is to provide a brief but comprehensive review of the full flow of information from the science part of the space weather chain, through the established geoelectric field interface, to the power engineering assessments. For this, we will review the status of scientific understanding in individual space physical and geophysical domains in the geoelectric field and GIC context. We will also provide descriptions of the links between the domains as they pertain to GIC. Further, we will quantify the links between science and engineering elements of the problem, and to complete the systems science view, we describe how the analysis continues on the power transmission system side. The power transmission system response analyses complete the GIC problem from its source in the solar corona to impact on the grid and its dependent critical infrastructure.

As an indication of the maturity of the GIC science and to facilitate systematic evaluation and monitoring of the progress in the applied sciences dimension of the field, we will assign "readiness levels" for individual elements in the space weather chain pertaining to GIC. To this end, we will leverage the work carried out by NASA Applied Sciences Program in the Earth sciences context. More specifically, the NASA Applied Sciences Program has instituted a nine-step Application Readiness Level (ARL) index to track and manage the progression and distribution of funded projects (see Figure 1). This index is an adaptation of a scale used by NASA for managing technology development and risk (Technology Readiness Levels, TRLs) and reflects three main tiers of project research, development, and deployment. In general, ARLs 1-3 encompass discovery and feasibility; ARLs 4-6 address development, testing, and validation; and ARLs 7-9 focus on integration of the application into an end user's decision-making activity. Here we adapt and introduce the ARL concept for space weather applications purposes. We will use the concept to quantify our capacity to transport information between different links in the chain of interacting processes from the solar corona down to upper mantle of the Earth and ultimately to engineering and operational implications of GIC.

In Figure 2, we indicate the links in the space weather chain pertaining to GIC. Figure 2 also displays the systems science view of GIC. In the figure, bold typeface "geoelectric field" indicates the interface between the science and engineering, and the engineering elements are indicated with red arrows and text. We use ARLs to describe our capacity to apply items in one link to generate information in the following link, i.e., our readiness to push information between the links. For example, ARL in the interface between Link B and Link C quantifies our capacity to take the geoelectric field and DC parameters of the system and then convert those into a GIC distribution within the system. It should be noted that the ARL assignments in Figure 2 and sections 


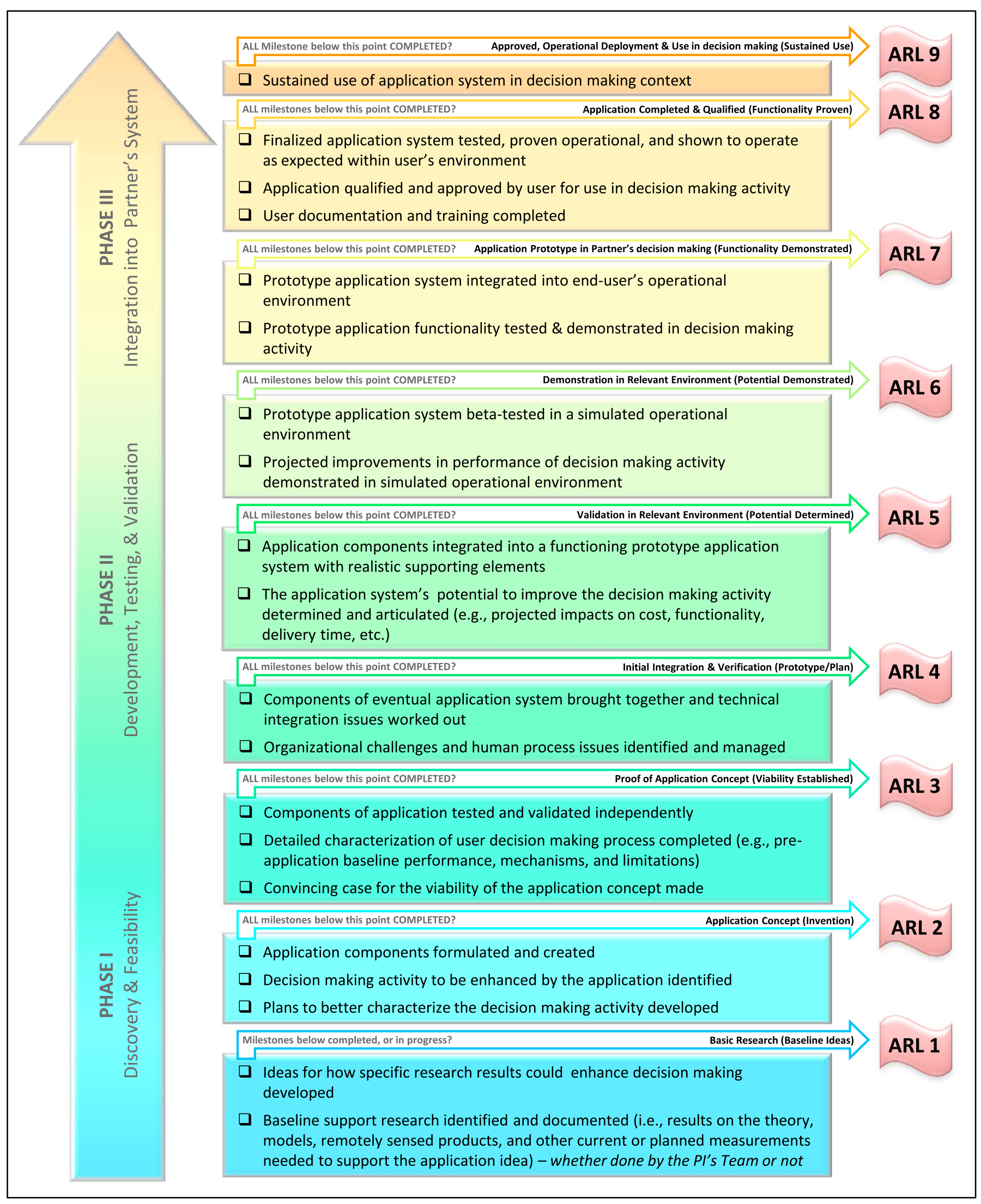

Figure 1. NASA Applied Sciences Program Applications Readiness Level (ARL) definitions. 


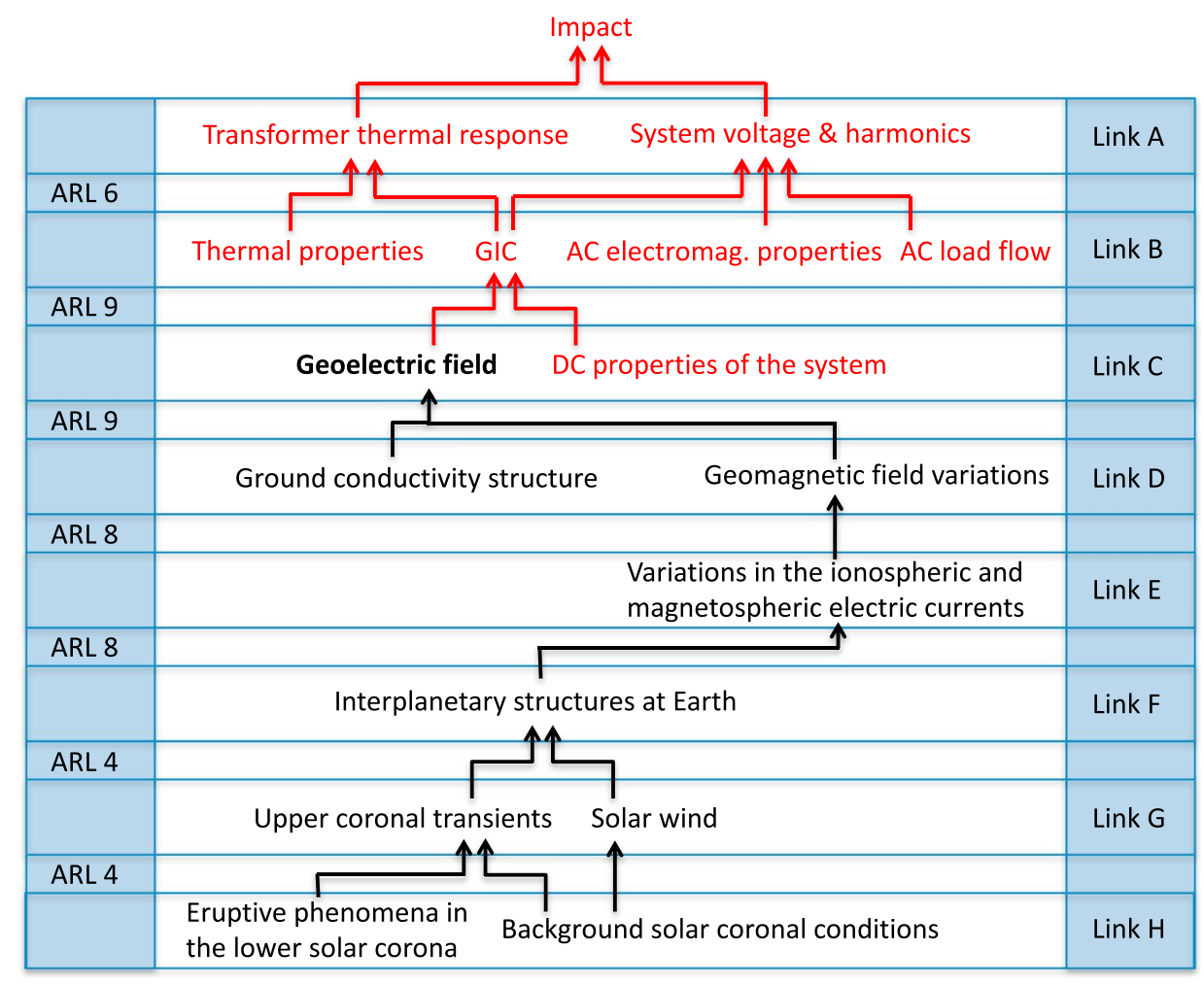

Figure 2. GIC systems science view and the links $(A-H)$ in the space weather chain pertaining to the problem. The arrows show the direction of propagation of information through the system and Applications Readiness Levels (ARLs) indicate our capacity to push information between the links. Black sections of the chart indicate the science components and red sections indicate the engineering components of the problem. Black bold typeface indicates the interface, geoelectric field, and between the science and engineering dimensions of GIC. Note that our ARLs assignments reflect maturity pertaining only to GIC-related applications. See Figure 1 for ARL definitions.

below pertain specifically to GIC-related applications. The maturity for pushing information between different links can be different for other types of applications.

ARLs, similar to TRLs, are not meant to measure the performance of individual models or tools but to quantify their readiness for use in decision-making context. It should also be noted that ARL 9 does not mean that "the job is done." While there may be ARL 9 applications that have been in sustained used in a specific decisionmaking context, there can be significant room for improving the quality and performance of those applications. This distinction between performance and application readiness should be kept in mind when considering the assigned ARLs in the sections below. We emphasize that assignments of specific ARLs reflect only our somewhat subjective views on the state of the GIC science and engineering. The ARL assignments should thus be considered as an introduction to the overall "readiness level" concept and a preliminary benchmark that will be adjusted over time as the wider discussion within the GIC and space weather communities takes place.

In sections 3.1-3.8, we will discuss the individual links in Figure 2 in more detail and provide arguments for assigning certain ARLs between the links. For clarity, we also explicitly indicate the types of sciences or engineering involved in the link and explain how the link pertains to the GIC problem.

\subsection{Link H: Eruptive Phenomena and Background Conditions in the Lower Solar Corona}

Sciences involved solar physics.

Significance to the GIC problem: lower solar corona phenomena are the ultimate driver of GIC. Full treatment of GIC and long lead time predictions require the capacity to understand and model dynamics in the solar corona.

While the solar atmosphere and eruptive events in it are driven from below by a variety of plasma physical processes such as the solar dynamo operating in the convection zone, we will start our discussion from 


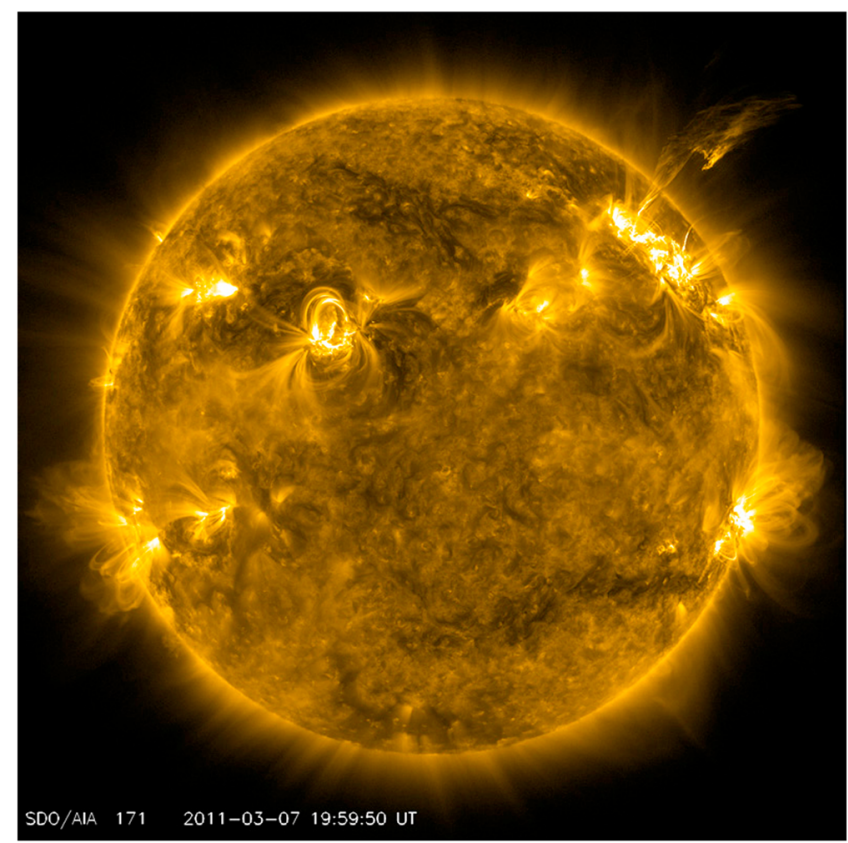

Figure 3. NASA Solar Dynamics Observatory image of the solar corona at $171 \AA$ A wavelength. Top right corner of the image shows an eruption propagating toward outer corona. Image was taken on 7 March 2011. the lower solar corona (Figure 3). When we make this choice, the lower solar corona is the domain that represents the first link in the space weather chain. Lower corona here indicates the "birthplace" of solar transients such as CME and is below about 2 solar radii. Coronal heating and the corresponding outflow of solar atmospheric charged particles, i.e., solar wind, together with buildup and release of magnetic energy in the corona are the fundamental physical processes that dictate the evolution of interplanetary structures that drive the magnetosphericionospheric response leading to terrestrial geomagnetic field variations and GIC. Correspondingly, the capacity to model and predict the background conditions and eruptive phenomena in the lower solar corona would allow the largest possible lead time GIC predictions. However, despite the major progress in remote solar imaging and physics-based modeling of the solar corona, the science is still relatively immature in terms of practical applications that could be used for GIC mitigation purposes. For example, we cannot yet satisfactorily predict the timing or size of solar eruptions.

\subsection{Link G: Upper Coronal Transients and Solar Wind}

Sciences involved solar physics and heliospheric physics.

Significance to the GIC problem: solar transients such as coronal mass ejections (CMEs) and solar wind structures such as stream interaction regions are the main drivers of geomagnetic events leading to GIC. Analysis and modeling of solar wind and solar transients can, in principle, provide 1-2 day lead time GIC predictions.

ARL to solve properties of upper coronal transients from the known properties of eruptive phenomena in the lower solar corona in Link H: 4. Rationale for the ARL assignment: per Figure 1, there are integrated modeling components readily available for ingesting information from the lower corona and photosphere to characterize corresponding dynamics in the upper corona. However, there is at this time no functional prototype available that would propagate the information to the GIC level.

While significant research efforts are underway to use physics-based models for initiation of transients in the lower solar corona and propagating information into the upper corona and interplanetary space [e.g., Tóth et al., 2007; Lionello et al., 2013], the state-of-the-art models that require time consuming case-by-case analyses carried out by solar physics experts are not yet ready for use in practical GIC assessments or forecasting. In some sense, the capacity to estimate solar wind conditions from remote solar photospheric observations is more mature, which is reflected in transitioning of one of the major solar wind models into operations at NOAA SWPC [Pizzo et al., 2011]. However, since predicting the detailed GIC-related dynamics of solar wind stream interaction regions remains a challenge and since CMEs are the driver of major and extreme GIC events [e.g., Borovsky and Denton, 2006; Huttunen et al., 2008; Kataoka and Pulkkinen, 2008], application readiness is constrained despite the operational capacity at NOAA SWPC.

Currently, the detailed kinematic analysis of solar transients starts once eruptions have left the "birthplace" in the lower solar corona and have propagated to the upper corona at about 2-30 solar radii and are imaged with remote sensing coronagraph instruments (Figure 4). Analysis of coronagraph observations and 


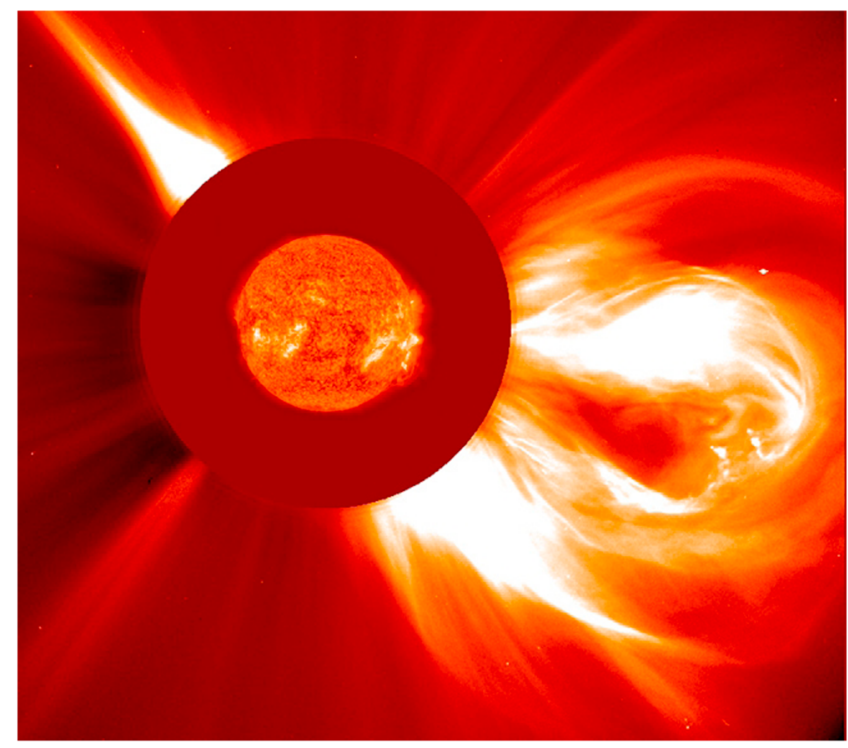

Figure 4. NASA/ESA Solar and Heliospheric Observatory (SOHO) Extreme ultraviolet Imaging Telescope instrument image of solar atmosphere at $304 \AA$ (center part of the image) and SOHO Large Angle and Spectrometric Coronagraph C2 coronagraph image of CME on 2 December 2003 (outer part of the image). derivation of transient properties allow usage of physics-based models that propagate the information through the heliosphere and to the orbit of the Earth. Much of our recent improved understanding in the kinematic evolutionary effects of transient has relied on triangulating the 3-D morphology through the use of multispacecraft techniques. While these triangulating techniques to model CMEs are often based on the expected structure under ideal conditions [e.g., Thernisien et al., 2009; Savani et al., 2009], they have proven to be of significant assistance to improve our understanding of the morphological features. This is true even though background heliospheric conditions often present a more complex scenario deforming transients away from ideal [e.g., Savani et al., 2010]. These types of analyses will be discussed further in the section below.

We note that while space-based coronagraph observations have dramatically improved our general space weather forecasting capacity, since observations are based on instrumentation on NASA STEREO and NASA/European Space Agency Solar and Heliospheric Observatory (SOHO) research missions, there is no guaranteed continuity for these critically important data [e.g., Vourlidas, 2015]. The lack of guaranteed continuity has a major impact also for long lead time GIC forecasts. Without knowledge about eruptions propagating in the upper solar corona, we cannot issue reliable early warnings about transients en route to Earth.

\subsection{Link F: Interplanetary Structures at Earth}

Sciences involved heliospheric physics.

Significance to the GIC problem: interplanetary structures impacting and interacting with the Earth's magnetosphere drive magnetospheric-ionospheric activity leading to geomagnetic activity and GIC. Geoeffective CMEs and solar wind stream interaction regions are the most significant interplanetary structures from the GIC viewpoint.

ARL to solve the properties of interplanetary structures at Earth from the known properties of upper coronal transients and solar wind in Link G: 4 . Rationale for the ARL assignment: while there are operational ARL nine interplanetary forecast products available, those do not yet have key information pertinent to the GIC problem. More complex modeling approaches have been developed but those have not entered systematic prototyping phase yet.

Major recent progress has been made in physics-based modeling and predicting the propagation of solar transients through the inner heliosphere. As mentioned in the section above, some of these models such as Wang-Sheeley-Arge-Enlil have been thoroughly validated and are already in operational use at NOAA SWPC [Pizzo et al., 2011; Millward et al., 2013]. However, the current models are used mostly to assess the locations in the heliosphere expected to be impacted by CMEs and estimate the arrival time of the transients at a variety of locations within the heliosphere such as Earth [e.g., Zheng et al., 2013]. The models used presently in real-time analyses do not incorporate information about CME internal magnetic field and have limited spatial and temporal resolution, which does not allow for the capture of steep boundaries or turbulent variations in the interplanetary plasma and magnetic field conditions. Consequently, these models are limited in their capacity to provide the detailed information about interplanetary plasma and magnetic field structures required for predicting GIC [Pulkkinen et al., 2009a]. Next generation models being developed target a more detailed description of the CME magnetic field and have a promise also for improved geomagnetic storm and 
GIC forecasts [e.g., Savani et al., 2015; Shiota and Kataoka, 2016]. We also note that since many of the historical extreme storms were cause by multi-CME events, inclusion of realistic CME-CME interactions [e.g., Liu et al., 2014] is an important goal in the future modeling efforts.

Since CMEs are believed to cause the most significant GIC, they are of special interest especially in the extreme events context. Consequently, we recognize that to facilitate progress in capturing the key CME processes pertaining to GIC, it may be worthwhile to attack the transient transport modeling challenge in separate components (see Kataoka and Ngwira [2016], for a similar idea in terms of magnetospheric-ionospheric response and $\mathrm{GIC}$ ). CMEs have a series of elements driving magnetospheric-ionospheric and geomagnetic activity. For fast CMEs, the initial impact is felt via shock wave formed ahead of the transient by superAlfvénic movement of the driver plasma. CME shock waves can drive sudden geomagnetic impulses and corresponding GIC that are felt globally [e.g., Kappenman, 2003]. The sheath region between the CME shock and driver plasma contains very turbulent magnetic field fluctuations that can also drive significant geomagnetic and GIC activity [Huttunen et al., 2008]. Finally, the main driver plasma and associated magnetic cloud "southward $B_{z}$ " powers a variety of currents systems throughout the magnetosphere-ionosphere system leading to the main phase of geomagnetic storms and GIC activity. From the GIC analysis and prediction viewpoint, we advocate the idea that in addition to a full physics-based approach that could capture all three key CME elements at once, one may also consider attacking individual CME elements separately. For example, present physics-based heliospheric models can already propagate shocks, providing tools for capturing the first CME element. Separate CME flux rope models such as Savani et al. [2015] provide tools for characterizing the "southward $B_{z}$ " element of CMEs. Development of models and tools that utilize remote sensing observations to capture CME sheath density and velocity characteristics are of great interest as well [e.g., Savani et al., 2013].

\subsection{Link E: Variations in the lonospheric and Magnetospheric Currents}

Sciences involved heliospheric physics, magnetospheric physics, and ionospheric physics.

Significance to the GIC problem: external electric current system variations drive the electromagnetic induction process in the Earth. A variety of electric current systems such as magnetopause, ring current, equatorial electrojet, and auroral ionospheric electric currents are known to drive the electromagnetic induction and GIC.

$A R L$ to solve variations of the ionospheric and magnetospheric currents from the known interplanetary structures at Earth in Link F: 8. Rationale for the ARL assignment: modern geospace model has been transitioned into operations to provide new GIC forecast products. The full suite of operational products is still under development and has not been in sustained use yet.

Perhaps the most significant recent progress in modeling space weather pertains to maturation and extended validation of both empirical and physics-based global magnetosphere-ionosphere models [e.g., Pulkkinen et al., 2013; Glocer et al., 2016]. The common approach to physics-based modeling uses a single fluid ideal magnetohydrodynamic (MHD) model coupled with ionospheric and inner magnetospheric modules. The global magnetospheric models can capture the physics of key electric current systems such as magnetopause current, ring current, field-aligned currents connecting the magnetosphere-ionosphere system, and auroral currents. Consequently, the space weather community has explored the usage of the latest generation of models for geomagnetic field perturbation and GIC prediction purposes [e.g., Pulkkinen et al., 2009b; Zhang et al., 2012]. The success of these global magnetospheric models is demonstrated by the recent transition of the University of Michigan's Space Weather Modeling Framework (SWMF) into operations at NOAA SWPC. The primary goal of the SWMF transition is to provide improved products to the power transmission system end users. Further, while much new work is required to better understand the limitations of such approaches, physics-based models have also been utilized in the latest extreme GIC event studies [e.g., Ngwira et al., 2014]. With the current heavy interest in extreme event assessment, one of the goals is that the combination of statistical and physics-based analyses will "converge" providing improved confidence for the estimated extreme event levels and occurrence frequencies.

Despite the significant progress in the field, the global magnetospheric and ionospheric electric current variations pertaining to GIC are very challenging to replicate accurately. This challenge is related to the two-faceted nature of GIC and geomagnetic storms: while the more predictable large-scale geomagnetic 
footprint of the storm is of global nature, the fluctuations in the geomagnetic signature that drive the geoelectric field can be highly complex and localized [e.g., Ngwira et al., 2015; Pulkkinen et al., 2015]. The highly dynamic nature of the storm time geospace system poses significant demands for reproducing the external electric current systems, and especially their fluctuations, in the right place at the right time and with correct amplitudes. Small displacements of the ionospheric electric current system can mean very large errors in the predicted local geomagnetic field variations that drive the geoelectric field and GIC. Also, it is possible that because some of the physical processes such as magnetic reconnection are not modeled self-consistently with modern single fluid ideal MHD, key electric currents such as the magnetotail current connecting to the ionosphere are not captured self-consistently either. Robust validation and improved understanding of limitations associated with the current physics-based magnetosphere-ionosphere models are thus important for advancing GIC research.

Also, purely observational specification of the global magnetospheric and ionospheric electric currents pertaining to GIC is challenging. While spacecraft constellations such as Cluster, Time History of Events and Macroscale Interactions during Substorms, Swarm (THEMIS), and Meteosat microwave sounder have the capacity to provide estimates of the electric current densities in space using the curlometer technique, observations are only pointwise or local at best [e.g., Ganushkina et al., 2015]. The AMPERE project, which uses the curlometer technique with the low-Earth orbit Iridium spacecraft constellation, is currently the only means to provide direct specification of global and large-scale ionospheric field-aligned electric currents [Anderson et al., 2000]. AMPERE supplemented with possible assimilation of global ground magnetic field observations and ionospheric radar information using methods such as AMIE [Richmond, 1992; Richmond et al., 1998] also provides a great opportunity for advancing the science of GIC.

\subsection{Link D: Ground Conductivity Structure and Geomagnetic Field Variations}

Sciences involved magnetospheric physics, ionospheric physics, and geophysics.

Significance to the GIC problem: geomagnetic field variations and local geological conditions in terms of conductivity structure from the surface down to the upper mantle depths are the key physical quantities that determine the geoelectric field driving GIC.

ARL to solve geomagnetic field variations from the known magnetospheric-ionospheric electric current structures in Link E: 8 . Rationale for the ARL assignment: modern geospace model has been transitioned into operations to provide new geomagnetic field variations and GIC forecast products. The full suite of operational products is still under development and has not been in sustained use yet.

Because GIC is a quasi-DC phenomenon, the computation of the instantaneous geomagnetic field variations by given external electric current systems is in principle a simple application of the Biot-Savart law expressed as

$$
\boldsymbol{B}(\boldsymbol{r})=\frac{\mu_{0}}{4 \pi} \frac{\int_{C^{\prime}} / \mathrm{d} \boldsymbol{l} \times \boldsymbol{r}^{\prime}}{\left\lfloor\boldsymbol{r}^{\prime}\right\rfloor^{3}}
$$

where the integration $C$ is performed over all electric currents $I$ in the system. Consequently, if magnetospheric-ionospheric currents are specified accurately, it is possible to compute accurately the corresponding external geomagnetic field variations on the ground [e.g., Rastätter et al., 2014]. This approach is used also in the operational implementation of the Space Weather Modeling Framework at NOAA SWPC. Solving for geomagnetic field variations generated by internal sources requires analysis of the geomagnetic induction problem, which in turn requires information about both the external source currents and the ground conductivity structure. The total geomagnetic field observed on the ground is the superposition of the external and internal components.

The most straightforward means to obtain information about the total geomagnetic field variations on the ground is to measure them directly. Geomagnetic field observations have been carried out for more than a century, and modern digital records are available since the 1970s. Further, observations are carried out globally by a number of international organizations and much of the collected data is available in real time. While geomagnetic field measurements cannot be used to forecast GIC, the observations together with geoelectric field modeling discussed below can elevate the situational awareness and provide means to trigger GIC mitigation actions. Perhaps most importantly, long historical geomagnetic field records 


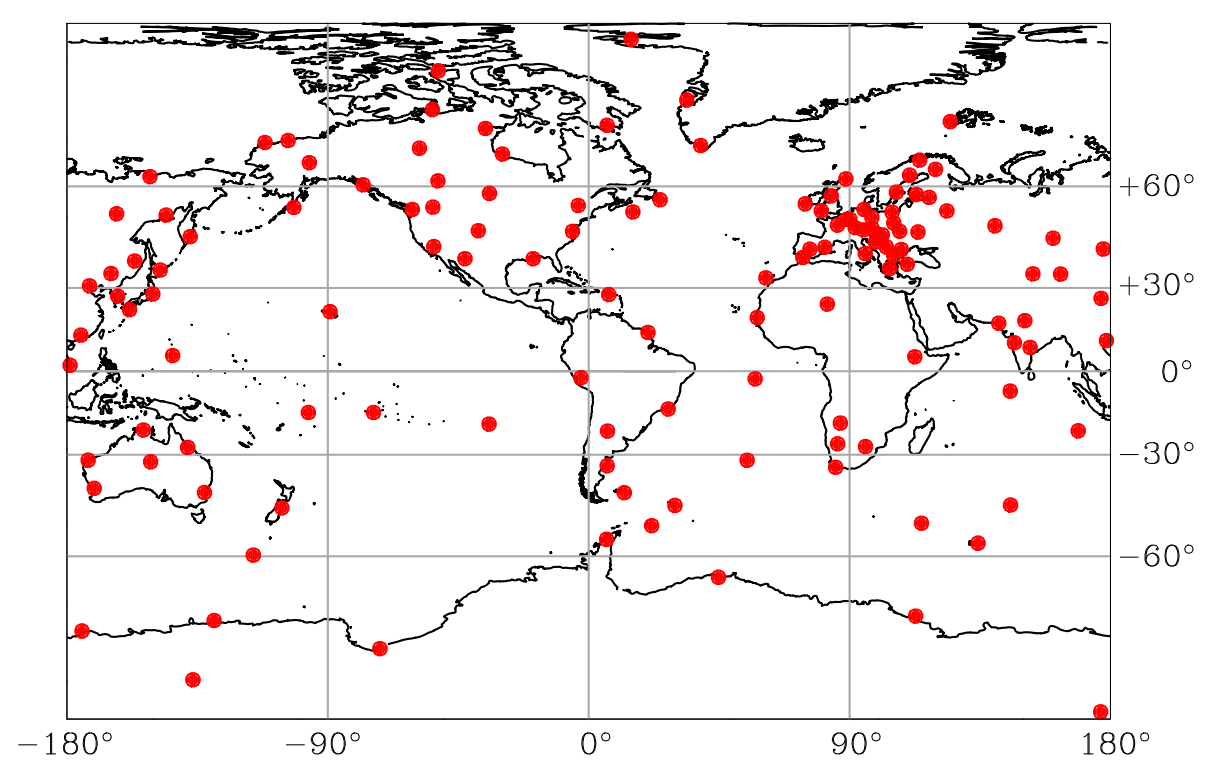

Figure 5. Map of permanent geophysical observatories providing historical and in some cases, real-time geomagnetic field measurements.

together with geoelectric field modeling allow statistical analyses of GIC and extreme event assessments [e.g., Thomson et al., 2011; Pulkkinen et al., 2012; Love et al., 2016a]. These statistical analyses currently provide the foundation for understanding the hazard GIC poses on power transmission systems, and the assessments are being used as a baseline for regulatory actions in the U.S. [North American Electric Reliability Corporation, 2016a].

The key challenge pertaining to geomagnetic field recordings is the spatial and temporal coverage of the geophysical observatories. In many locations such as the contiguous U.S., the spatial density of permanent geophysical observatories is not high and it is recognized that given the storm time complexity of the field variations, more observatories are needed to allow sufficient spatial coverage for space weather applications (see Figure 5). Optimizing the distribution of possible new geomagnetic recording stations for GIC applications is an outstanding issue that calls for additional investigations.

As will be discussed in more detail in the section below, the ground conductivity structure from the surface down to the upper mantle of the Earth dictates how the electromagnetic field and GIC on the surface of the Earth responds to external magnetospheric-ionospheric electric current variations. This geophysical component of the GIC problem may also be the most challenging. In many situations, poor knowledge about local ground conductivity and the corresponding electromagnetic response can be the dominant source for GIC modeling uncertainty. While there has been a large collection of deep-Earth electromagnetic sounding campaigns across the globe, the surveys have been motivated by basic scientific research and not GIC applications. Also, the geological structures can be highly variable making a survey directly applicable only to that specific location [e.g., Bedrosian and Love, 2015]. It is thus of major interest from the GIC standpoint to extend the electromagnetic sounding campaigns to cover all key areas of GIC interest. As an example, it is highly desirable to complete the USArray magnetotelluric (MT) survey and corresponding analyses that can extract three-dimensional information about the high spatial resolution $(\sim 70 \mathrm{~km})$ ground conductivity structures across the contiguous U.S. [Williams et al., 2010].

\subsection{Link C: Geoelectric Field and DC Properties of the System}

Sciences involved geophysics and power system engineering.

Significance to the GIC problem: geoelectric field induced on the surface of the Earth is the primary physical quantity driving GIC, and mapping from the geoelectric field to GIC is determined by the DC properties of the technological system of interest. 
ARL to solve for the geoelectric field from the given ground conductivity structure and geomagnetic field variations: 9. Rationale for the ARL assignment: geoelectric field products that use observed geomagnetic field variations and one-dimensional (1-D) ground conductivity models have been used in applications context for several decades. The modeled geoelectric fields have been used in situational awareness applications and hazards assessments by the end users and thus fulfill the "sustained use in decision-making context" criteria.

The so-called "plane wave method" introduced by Cagniard [1953] has been the workhorse of much of the GIC research and applications for decades. The plane wave method assumes that the magnetic field (external geomagnetic field variations) from the magnetosphere-ionosphere source can be approximated as a planar wave and that the ground conductivity can be approximated as a one-dimensional structure varying only as a function of depth. Importantly, the plane wave method has been shown to be a sufficiently accurate approach in many situations if both local geomagnetic field variations and effective 1-D ground conductivity are known to a good approximation [e.g., Trichtchenko and Boteler, 2004; Viljanen et al., 2006; Ngwira et al., 2008; Wik et al., 2008; Pulkkinen et al., 2010]. Note the emphasis on "effective" 1-D conductivity. True ground conductivity variations are usually three-dimensional (3-D) and consequently applied 1-D models should be interpreted only as effective approximations that allow reproducing the corresponding GIC satisfactorily [see also Beggan, 2015]. We also note that although most of the plane wave applications have used the frequency domain formulation, time domain formulations have been developed and applied [e.g., Viljanen and Pirjola, 1989; Marti et al., 2014].

Since the true ground conductivity structure is typically 3-D, despite the success of the 1-D plane wave method used in the past, the natural next step in GIC research is to keep moving toward usage of 3-D electromagnetic induction methods and models [see, e.g., Simpson, 2011; Püthe et al., 2014; Samimi and Simpson, 2016]. For example, realistic treatment of the coast effect that amplifies the geoelectric field in coastal regions due to lateral conductivity gradients cannot be captured with 1-D models [e.g., Pirjola, 2013]. Investigations that use a range of realistic model parameters for the coast effect and related GIC flow analyses can illuminate the general importance of inclusion of the 3-D effects in space weather analyses and do not necessarily require representation of any specific local geological conditions. However, the use of 3-D induction models in direct applications will require an accurate representation of the 3-D ground conductivity. Detailed 3-D ground conductivity models are not yet readily available for many regions, which limits immediate usage of 3-D induction models in GIC applications.

There is a way to "bypass" the need for any specific ground conductivity models in GIC studies and applications. The geoelectric field can be computed from the ground magnetic field variations by using empirically derived surface impedance tensors [e.g., Bedrosian and Love, 2015]. Mathematically, this approach is expressed as

$$
\boldsymbol{E}(\omega)=\frac{1}{\mu}\left[\begin{array}{cc}
Z x x & Z x y \\
Z y x & Z y y
\end{array}\right] * \boldsymbol{B}(\omega)
$$

where $\mu$ is the magnetic permeability, $\boldsymbol{E}$ is the horizontal geoelectric field, $\boldsymbol{B}$ the horizontal ground magnetic field, and $Z$ the surface impedance tensor components. In equation (2), all quantities are expressed in the frequency domain. Following the standard magnetotelluric (MT) method, temporary instrument installations can be deployed to measure local $\boldsymbol{E}$ and $\boldsymbol{B}$ from which the impedance tensor can be derived. Importantly, if the full impedance tensor can be derived, 3-D effects are included in the operator. If a good-quality impedance tensor can be derived, local ground magnetic field measurements for any time period can be used to compute the geoelectric field via equation (2). Consequently, local MT surveys may be the optimal approach for obtaining good local models for mapping the observed geomagnetic field variations into the geoelectric field. However, while many surveys have been carried out across the globe and some arrays such as the nine permanent MT stations in South Africa have been deployed specifically for GIC research purposes, MT survey data are not yet available for many regions of interest. In North America, the USArray project and other surveys have covered only parts of the U.S. and Canada (Figure 6) [Schultz, 2010]. Further work is also needed to better understand how the local geoelectric field obtained via equation (2) should be applied in GIC computations. As will be shown below, GIC senses the geoelectric field in the length scales integrated over the transmission lines segments, which are typically 


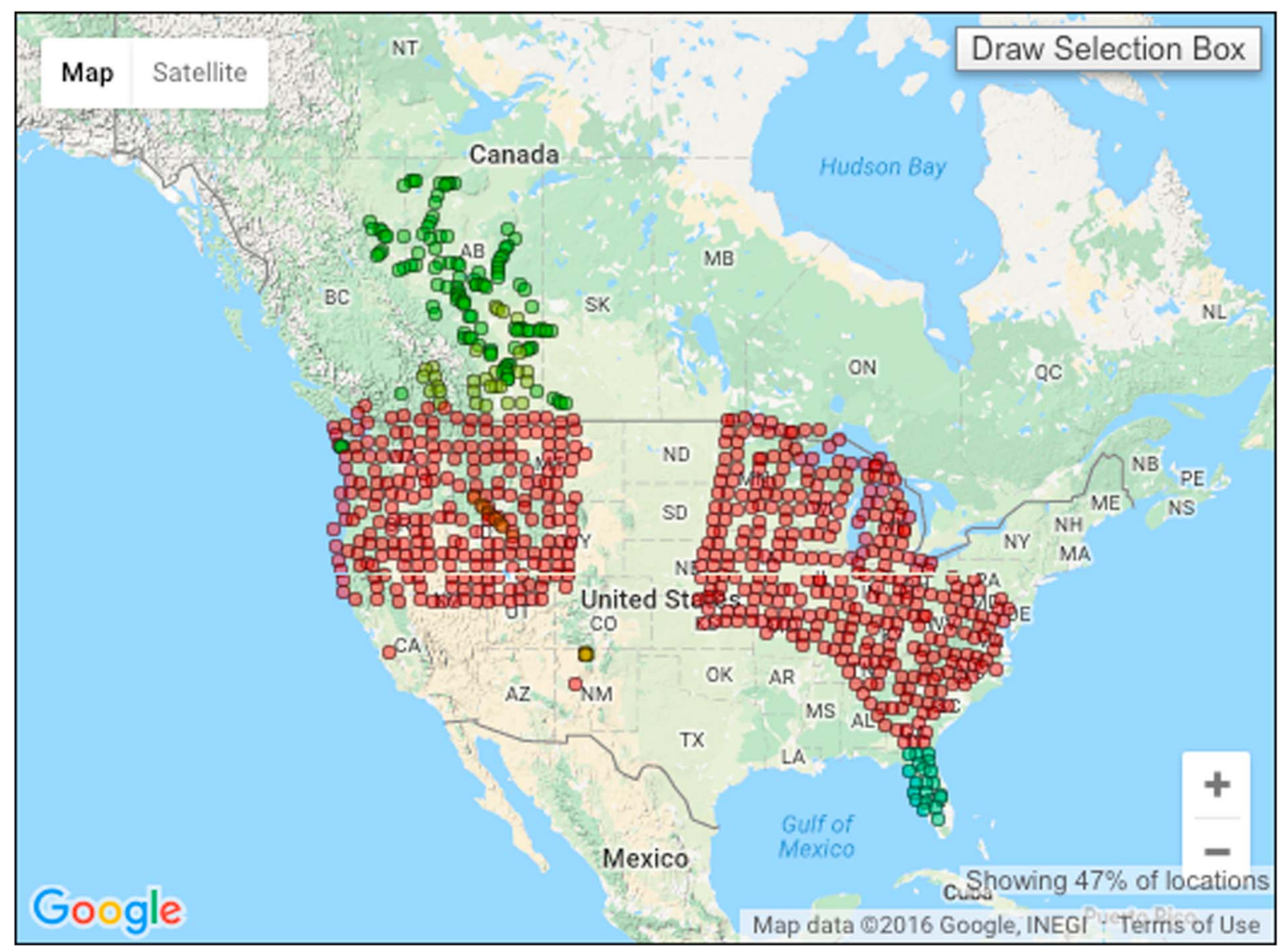

Figure 6. Map of North American locations covered by temporary MT installations that have data and empirical impedance tensors are available in the Incorporated Research Institutions for Seismology database at http://ds.iris.edu/spud/emtf.

of the order of $100 \mathrm{~km}$. Local representation of the geoelectric field via empirical impedance tensors that includes not only local inductive 3-D structures but also possible local galvanic effects may not be representative of the field at $\sim 100 \mathrm{~km}$ scales (see, e.g., Bedrosian and Love [2015, Figure 2] for showing significant variations between nearby MT stations). The key consideration here is that if the computed geoelectric field is a very local representation, one needs to be careful in applying the same field over large areas in GIC calculations.

As the interface between the engineering and science communities, the geoelectric field is also the interface for hazard assessments and extreme event studies. Consequently, as was indicated in section 3.5, one of the most pressing challenges is to characterize the geoelectric field during extreme storm events. Extreme event studies will require characterization of the local ground geomagnetic field and local geological conditions mapped into the geoelectric field [e.g., Boteler, 2001; Pulkkinen et al., 2012; Love et al., 2016a, 2016b; Nikitina et al., 2016]. Importantly, extreme geoelectric field scenarios need to specify both the spatial and temporal evolution of the geoelectric field to allow detailed engineering assessments. The recent discovery of localized extreme geoelectric field enhancements calls for improved understanding of the underlying magnetospheric-ionospheric physics and inclusion of relevant spatial scales in extreme event analyses [Ngwira et al., 2015; Pulkkinen et al., 2015]. Also, local geological features can give rise to localization of the geoelectric fields as seen in the maps by Bedrosian and Love [2015]. Different spatial scales have different implications for the performance of high-voltage power transmission systems during extreme storms [North American Electric Reliability Corporation, 2016a] and thus characterization of the extreme geoelectric fields at appropriate spatial scales is a significant ongoing science challenge. 
The geoelectric field is mapped into GIC using an electromagnetic characterization of the conductor system of interest. Since geomagnetic field fluctuations having the most power in periods of 1-1000 s are a low-frequency phenomenon from the power transmission system 50 or $60 \mathrm{~Hz}$ perspective, the mapping can be carried out to a good approximation as a DC problem. Exact treatment would require considering also the reactive components of the transformer and transmission line impedances. However, the reactive component is a second-order effect operating only at the highest GIC frequencies, and it can be shown that accounting for the interaction between the transformer windings and GIC tends to reduce the GIC amplitudes [Boteler and Bradley, 2016; see also Oyedokun, 2015]. Consequently, DC approach represents a conservative treatment, which should be noted especially in the hazards assessments context.

For the DC treatment of GIC flow, DC properties such as conductor resistance, substation grounding resistance, and topology need to be specified. GIC is then solved individually for each time step with the specified geoelectric field using DC approach. In the case of discretely grounded electric power transmission systems, GIC can be modeled either as an impedance network or as an admittance network (see Boteler and Pirjola [2017], for a review). The two methods are mathematically equivalent. In the impedance network approach, the model inputs are the voltages obtained by integrating the electric field along each transmission line, while in an admittance network approach, these voltages are converted to equivalent current sources. The impedance network approach is conceptually simpler, but the admittance network approach is computationally more efficient for large networks. For simplicity, impedance network approach is presented below.

In the impedance network approach, GIC can be solved using the following set of DC equations [Lehtinen and Pirjola, 1985]:

$$
\begin{gathered}
\boldsymbol{I}^{e}=\left(\mathbf{1}+\boldsymbol{Y} \boldsymbol{Z}^{\boldsymbol{e}}\right)^{-1} \boldsymbol{J}^{e} \\
Y_{i j}= \begin{cases}\frac{-\frac{1}{R_{i j}}}{\sum_{k \neq i} \frac{1}{R_{i k}}} & i \neq j\end{cases} \\
J_{j}^{e}=\sum_{i \neq j} \frac{V_{i j}^{0}}{R_{i j}}
\end{gathered}
$$

where $\boldsymbol{I}^{e}$ is the GIC flowing through the transformer groundings, $R_{i j}$ are the transmission line resistances between transmission nodes $i$ and $j, Z_{i j}{ }^{e}$ is the grounding impedance matrix, and

$$
V_{i j}^{O}=\int_{i}^{j} E \cdot d s
$$

where $\boldsymbol{E}$ is the horizontal geoelectric field and the integration is carried out between the transmission system nodes $i$ and $j$. From equations (3)-(6) it is clear that not only the accuracy of the geoelectric field but also the accuracy of the DC parameters determine the GIC modeling accuracy. The resistances of high-voltage transmission lines are well known. Resistance values for transformer windings are also available but are not generally used in power system studies, so the values can be difficult to find. However, the largest source of uncertainty in the DC description is the values for the substation grounding resistances $Z_{i j}{ }^{e}$. It is uncommon to measure actual DC grounding resistances and that of the surrounding soil at substations, and conditions vary significantly between different locations making the usage of generalized values impractical. Systematic surveys of high-voltage power transmission DC grounding resistances are thus one of the areas that will allow reduction in GIC modeling inaccuracy. Finally, from equation (6) that shows the voltage source as the integral, i.e., average, of the field over the path of the transmission line, it is clear why the spatially averaged geoelectric field between the system nodes, not the local geoelectric field, is the key target in GIC investigations.

\subsection{Link B: GIC, Transformer Thermal Properties, Electromagnetic Properties, and AC Load Flow}

Sciences involved power system engineering.

Significance to the GIC problem: GIC flow and power transmission system AC properties determine the system impact. 
ARL to compute GIC distribution from the known geoelectric field and DC parameters of the system in Link C: 9. Rationale for the ARL assignment: the DC GIC computation methods discussed in the section above have been used in applications context for several decades. The modeled GIC have been used in situational awareness applications and hazards assessments by the end users and thus fulfill the sustained use in decision-making context criteria.

This step is probably the best developed component of GIC research. There are a number of well-validated and operationally applied methods such as the one indicated in equations (3)-(6) to compute GIC. As only a relatively straightforward DC formulation of the problem is needed, computation of GIC may also be mathematically the simplest step in the chain. However, the accuracy of the DC description of the system and the geoelectric field is critically important for obtaining accurate GIC distribution in the system. Methods for computing GIC both in discretely grounded systems such as power transmission grids and continuously grounded systems such as buried pipelines have been developed [Boteler, 1997; Boteler and Pirjola, 2014, 2017].

Due to the long line lengths and low line resistances, the primary GIC impact takes place at the high-voltage portion of the electric power transmission system. As a rule of thumb, systems operating at $200 \mathrm{kV}$ and above are vulnerable [North American Electric Reliability Corporation, 2013]. In the high-voltage power transmission systems, the impact is due to an interplay between quasi-DC GIC and regular AC transmission: GIC causes the so-called half-cycle saturation of transformers that leads to a series of secondary effects [e.g., Molinski, 2002]. The most important secondary effects are (1) transformer heating and possible damage due to stray AC magnetic flux from the saturated core, (2) deviation from the quasi sinusoidal forms of the electric current and voltage in the system, i.e., generation of harmonics, and (3) significant increase in the transformer magnetizing current leading to a change in the balance between "real" power available for serving the system load and imaginary or "reactive" power fluctuating within the transmission system itself (for a conceptual introduction to power engineering terminology, see, e.g., Meier [2006]). Importantly, reactive power and the system voltage are linked. Consequently, major changes in reactive power can lead to system voltage instabilities and in the worst cases to a voltage collapse (blackout). According to the latest assessments by the NERC Geomagnetic Disturbances Task Force, voltage collapse associated with GIC-driven reactive power changes is the most likely scenario for bulk transmission system impact during extreme storms [North American Electric Reliability Corporation, 2012]. System harmonics, in turn, can cause protective relay tripping and drop lines from service. The famous March 1989 Hydro-Quebec blackout was caused by a combination of harmonics-driven relay trippings and system voltage collapse [e.g., Bolduc, 2002]. The regional blackout in Malmo Sweden during the October 2003 storm was caused by a relay tripping that dropped a single critical line from service [Pulkkinen et al., 2005].

While there is a documented heating-related transformer damage from the New Jersey Saleem power station during the March 1989 storm and in several South African power transformers from the October-November 2003 storms [Gaunt and Coetzee, 2007], based on the latest assessments, wide-scale immediate and permanent damage of a large number of transformers is unlikely [e.g., North American Electric Reliability Corporation, 2012; Royal Academy of Engineering, 2013]. One key reason for this thinking is that the high-amplitude GIC pulses are fairly short lived during major and extreme storms. Due to transformer thermal inertia, most GIC pulses do not last long enough to cause a possibly catastrophic elevation in the transformer hot spot temperatures. However, we note that the extent of the risk associated with heating-related permanent damage of transformers is still being debated (see, e.g., National Research Council [2008], for an alternate view and University of Cambridge, Center for Risk Studies, 2016). The U.S. Federal Energy Regulatory Commission's Phase II geomagnetic disturbances standard [Federal Energy Regulatory Commission, 2015] that was passed September 2016 will require all U.S. transmission operators having systems $200 \mathrm{kV}$ and above to carry out transformer heating analyses. Once the transmission system operators have carried out these analyses, we will be able to achieve at least partial closure to the debate.

To assess the half-cycle saturation-generated GIC effects, the AC properties of the electric power transmission system need be known. The key AC characteristics are transformer thermal properties, system electromagnetic properties, and AC load flow description that are associated with effects (1), (2), and (3) above, respectively.

Observational GIC data are important not only for validating the modeling but also in some cases to derive the models [e.g.,McKay, 2004]. We do note that as seen via equations (3)-(6), the GIC signal carries 


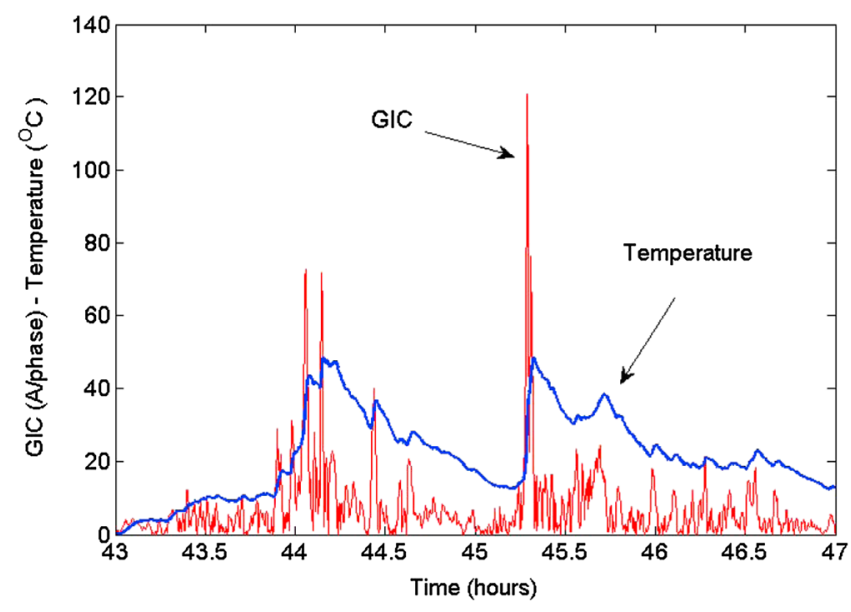

Figure 7. Sample transformer tie plate temperature calculation. Blue trace is incremental temperature and red trace is the magnitude of the GIC/phase. From Marti et al. [2013]. information about both the geoelectric field and system DC properties. Consequently, it can be challenging to separate these two factors directly from GIC observations unless DC properties of the system or local geological conditions are known accurately. In other words, there is an inherent ambiguity associated with the interpretation of the GIC signal. However, since GIC observations measure directly the quantity impacting system performance, such observations are the ultimate trigger of mitigation actions. Consequently, measurements are becoming increasingly commonplace across the globe. One example is the U.S. Electric Power Research Institute's SUNBURST network which has a rapidly expanding number of GIC observation stations [Electric Power Research Institute, 2011].

GIC data have commercial and physical security concerns in terms of GIC amplitudes and substation location information. Despite these sensitivities and inherent ambiguity in terms of interpreting the signal, GIC observations have great value for both scientific and engineering communities. Novel discoveries and progress can be made by providing public domain access to data, allowing for application of novel analysis techniques from a larger group of experts. An establishment of a GIC repository for scientific research purposes would help facilitate those new discoveries. The repository could be established in close collaboration with the industry and would include data from a number of stations with wide geographical coverage for special geomagnetic storm events of interest. In the same context, we encourage GIC recording procedures that will allow optimal use of the data in scientific analyses: (1) record GIC with $1 \mathrm{~s}$ or higher temporal cadence; (2) have sensitivity of $0.1 \mathrm{~A}$ or better and dynamic range over several hundreds of amperes; (3) include polarity of the current in the recordings; and (4) carry out and store recordings continuously and with GPSsynchronized timestamps.

We note that in most cases, GIC data that are recorded only for instances when the current exceeds a certain threshold are not optimal for detailed scientific analyses. Also, temporally continuous and extended records with accurate timestamps facilitate joint analysis with other scientific data such as local geomagnetic field recordings.

\subsection{Link A: Transformer Thermal Response, System Voltage and Harmonics}

Sciences involved: power system engineering.

Significance to the GIC problem: determining the response of the system in terms of transformer thermal response, system voltage, and harmonics is the final engineering end link of the GIC problem. Understanding the engineering implications allows quantification of the impact.

ARL to quantify the impact from known GIC and system AC characteristics: 6 . Rationale for the ARL assignment: while commercial tools are available for GIC-related power flow calculations, this is not the case for the transformer heating and harmonics assessments. Voltage stability, transformer heating, and harmonics assessments have so far been carried out mostly by utility research departments on case-by-case basis.

There has been significant recent progress in developing power engineering tools that allow not only computation of GIC from the known system DC characteristics and geoelectric field but also carrying out electric power flow computations for system voltage stability assessments. Also, transformer thermal models are starting to become available allowing users to determine if given GIC can cause permanent damage to the transformers. However, harmonics considerations are not yet possible with commercially available tools and utilities that have carried out the analyses relied on assessments within the companies' research departments. New analysis tools need to be developed, validated, and made available for the engineering 

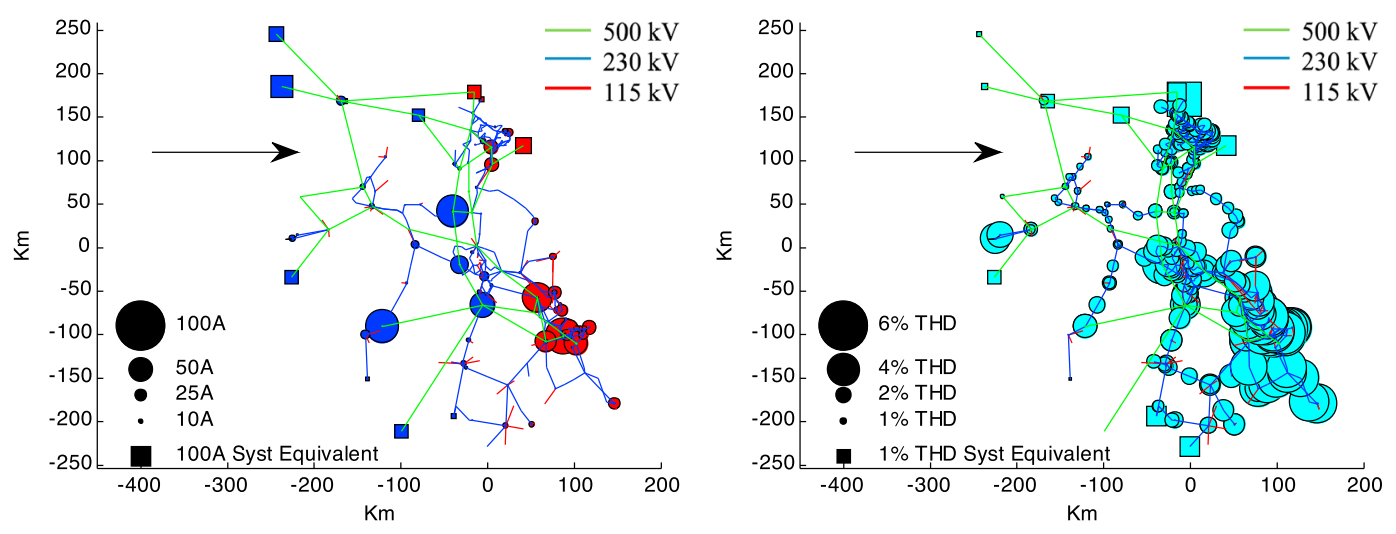

Figure 8. (left) GIC flows in Dominion Virginia Power's network for a 100 year storm. Transformers experience different amplitudes of GIC. The edges of the system tend to be critical locations, i.e., locations prone to experience larger amplitudes of GIC. (right) The resulting voltage THD. Harmonics propagate throughout the system and therefore do not exhibit the same focused critical locations. Significant voltage distortion can also occur at places where GIC is relatively low. From Bernabeu et al. [2015].

community to allow wider harmonics impact analyses. We discuss all three types of analyses associated with the three effects identified in section 3.7 more in detail below.

Effect (1) in section 3.7 is associated with analysis of transformer thermal response. Excessive and possibly localized (hot spot) heating caused by stray AC magnetic flux from the transformer core can lead to permanent damage of transformer components, which in turn can require moving equipment from service. Modeling the thermal response requires a transformer thermal model that is then used together with GIC information. In these analyses, both GIC amplitude and temporal evolution are critically important. Transformer thermal response time is typically of the order of minutes, which means that if an isolated GIC pulse or a few such pulses last only some tens of seconds, there is no time for substantial heating. On the other hand, long-duration and large-amplitude GIC can lead to excessive transformer heating. Figure 7 illustrates the correspondence between GIC and transformer hot spot temperatures using one of the thermal models that are starting to become available for GIC impact analyses. That said, thermal models are needed for individual transformers, and at this time the validation level of the models is not high. Comparisons between thermal models and actual observed transformer thermal responses are thus needed. There is also a need for commercially available software for carrying out the thermal analyses. For more detailed discussion on the thermal assessments, see Marti et al. [2013] and North American Electric Reliability Corporation [2016b].

Effect (2) in section 3.7 is associated with harmonics analysis. Under half-cycle saturation, transformers inject even and odd harmonics into the system. Excessive harmonic distortion can compromise the performance of protection and control systems. In a nutshell, harmonic distortion was the primary root cause of the 1989 Hydro-Quebec blackout; protection systems tripped critical reactive compensation equipment, leading to a voltage collapse.

Harmonic studies can be performed in the time domain (electromagnetic transient programs such as Electromagnetic Transient Program and Power Systems Computer-Aided Design) or in the frequency domain. Typical harmonic studies can be performed using a reduced model of the power system (just a few buses in size) and involve only a few of harmonic injections. Analyzing the impact of harmonics during geomagnetic storms poses some interesting challenges. First, the wide-area nature of a storm implies that there will be multiple harmonic injections. In essence, each saturated transformer becomes a harmonic source. Second, harmonics can propagate throughout the power system. As a result, to determine the total harmonic distortion (THD) in the system, a wide-area harmonic load flow model that considers multiple harmonic sources and the propagation of harmonics is needed. Figure 8 shows an example of geomagnetic storm harmonic load flow analysis for a transmission owner within PJM Interconnection [Bernabeu et al., 2015]. The figure illustrates that the location with maximum GIC amplitude does not necessarily coincide with the largest harmonic distortion.

Performing a system-wide harmonic load flow analysis with existing tools requires significant effort and certain level of expertise to tailor and manipulate multiple modeling tools. Given the critical role of harmonics in 
the overall geomagnetic storm risk assessment, there is a need to integrate wide-area GIC-driven harmonic load flow analysis into commercially available software.

Finally, effect (3) in section 3.7 is associated with electric power flow analyses. Large changes in the reactive and real power balance can cause system voltage fluctuations, and excessive changes in reactive power can ultimately lead to a system voltage collapse. Modeling the power balance in the system is carried out by the so-called power flow calculations that numerically model the behavior of electric power in an interconnected system. Power flow calculations that are a standard tool used by the power transmission industry assume known real and reactive power at the system load buses and known real power and voltage amplitude at the generator buses [see, e.g., Meier, 2006]. The GIC effect enters the analysis via reactive power that is adjusted based on the known GIC flow through the transformers. Consequently, GIC distribution throughout the system needs to be known. The computations then provide voltage angle and amplitude in the system. It is also important to note that the instantaneous GIC distribution is used instead of time series as in the thermal analyses. This is due to the fact that from the $60 \mathrm{~Hz}$ (or $50 \mathrm{~Hz}$ ) power flow viewpoint, GIC operating at millihertz range is a good approximation DC phenomenon.

The effects (1)-(3) discussed above are now fairly well understood and pertain mostly to high magnitudes of GIC flowing through high-voltage power transformers. "High magnitude" is a system-dependent quantity, but as a rough rule of thumb, only GIC well above $10 \mathrm{~A}$ are considered potentially significant. For example, the proposed Federal Energy Regulatory Commission (FERC) standard [Federal Energy Regulatory Commission, 2015] sets the thermal screening criterion at $75 \mathrm{~A}$ per phase. However, there are a number of reports [Forbes and St. Cyr, 2008; Forbes and St. Cyr, 2010; Schrijver and Mitchell, 2013; Gaunt, 2014] where researchers have indicated that also lower levels of GIC can have an impact on the performance of the power transmission system. These works are under ongoing debate, and the causal connections associated with the findings are not yet well defined. If there is a true connection to low levels of GIC, this would constitute a transition from current thinking of GIC as only a high-impact, low-frequency phenomenon to considering the phenomenon as a background that continuously and gradually degrades components and the performance of the system. A related open engineering question pertains to the debate about immediate failure versus possible storm-driven premature aging of transformers [e.g., Gaunt, 2014]. The idea that a damaged transformer does not necessarily fail during the storm but only afterward adds another layer of complexity to impacts assessments. To better understand these possible additional aspects of GIC would entail more work.

\section{Impact}

The series of Links H-A discussed above constitute the full space weather-related systems science chain extending from the solar atmosphere down to the upper mantle of the Earth and ultimately to the engineering impacts of GIC. Importantly, an interdisciplinary approach including space sciences, geophysics, and engineering with interfaces between the disciplines is necessary for quantifying the GIC problem. In our case, the interface between the science and engineering is the geoelectric field (see Figure 2 ) and Links A-C describe the engineering steps that allow determining how GIC influences the performance of the high-voltage power transmission system. Link A provides quantification of the ultimate GIC "impact" in terms of the system response.

Quantification of extreme event likelihoods and the corresponding system response is critical in understanding the risk space weather poses on power grids and society. Indeed, as was discussed in section 2.4 , it must be understood that risk, i.e., the possibility of adverse effects, is determined not only by the natural hazards themselves but also by the exposure and vulnerability to these hazards [see, e.g., Cardona et al., 2012]. In our application, the vulnerability is highly dependent on the engineering details of the system exposed to the hazard thus underscoring the importance of full flow of analysis from the science into engineering. We cannot understand and quantify the risk without a carefully coordinated multidisciplinary approach.

In this work, we have focused on GIC impacts on the high-voltage power transmission systems. While GIC is the primary impact on the power grids today, there are possible additional mechanisms by which space weather can influence the performance of future power transmission systems. As a part of moving toward implementation of "smarter grids," new technologies will become increasingly widespread. An example of such technologies is the so-called phasor measurement unit (PMU) that measures the voltage and current of the system with high accuracy at the system nodes. The key idea is that the information provided by PMUs offers a significant improvement in monitoring, control, and protection of the power systems, and it is expected that these 
devices will permeate the future grid. However, PMUs use common timing that is obtained via GPS, which is known to have space weather vulnerabilities. More specifically, GPS timing information can be degraded during major storms and consequently lead to possible problems in keeping the PMUs synchronized.

Increasing contributions of electric power generation from renewable sources may pose novel challenges. For example, offshore wind generation and corresponding transmission links can be exposed to the electromagnetic induction "coast effect" mentioned in section 3.6. Consequently, interdisciplinary studies could identify new emerging and rapidly spreading grid-related technologies and assess the corresponding potential vulnerabilities. While we have made major progress in understanding the science and engineering of GIC, it would be too optimistic to claim that all key challenges associated with space weather and electric power transmission are solved and fully under control. One of the lessons from history is that as our technology advances, we introduce new ways for space weather to influence our daily lives.

\section{Discussion}

The understanding of the threat that GIC poses on the high-voltage power systems has evolved over the past few years, and it is now widely believed that the most likely consequence of a major or extreme geomagnetic storm, if not mitigated, is a widespread system voltage collapse instead of permanent damage to a large number of transformers. However, the problem cannot be ignored. Widespread voltage collapse and a corresponding blackout are a very severe event that can impact the lives of millions of people and can lead to significant socioeconomic losses. Consequently, appropriate procedures and safeguards need to be in place for mitigating possible widespread impacts. In the U.S., the ongoing FERC geomagnetic disturbance standards process and significant components of the National Space Weather Strategy and National Space Weather Action Plan are designed to do exactly that. As a key element of these actions, scientific research on the topic needs to continue and the work needs to maintain direct links to the power engineering dimension of the problem.

The key challenge to scientists is to specify the spatiotemporal evolution of the geoelectric field, in the past, present, and future, and especially under extreme event conditions. The geoelectric field is the interface between the engineering and science disciplines, and improved information about the field under extreme conditions can be used to better understand the general nature of the hazard via power grid vulnerability assessments. Improved understanding of the future geoelectric fields, in turn, allows implementation and utilization of advanced mitigation actions helping to "weather" the major storms. Thankfully, we have come a long way in understanding the nature of the geoelectric field. We have a basic understanding of the core physical domains and processes in the space weather chain extending from the solar corona down to the upper mantle of the Earth involved in driving the geoelectric field. We also understand the linkage between the space weather phenomena and long conductor systems on the ground, i.e., how the geoelectric field generates GIC. Further, we understand the key engineering implications of GIC. One of the true breakthroughs in GIC science has been the full acknowledgment of the systems science aspect of the problem - the whole can be understood only by understanding the individual subdomains and the connections between them. The systems science approach is reflected especially in how the research community has opened new technical communications between disciplines: magnetosphere-ionosphere experts now work with geophysicists and geologists, scientists work with power engineers, and many different disciplines are represented in efforts such as the LWS Institute GIC Working Group.

Despite great progress, major challenges remain. For example, we have a very limited understanding of the upper limits for geoelectric field amplitudes. What is the worst that can occur? Major theoretical analysis and modeling efforts are needed to answer the question. Additionally, our capability to predict the geoelectric field and GIC remains very limited. This is especially true for long lead time predictions, which in the space weather context is of the order of 1-3 days. Our capability is hindered by the fairly immature state of the first-principle solar and heliospheric analysis and modeling. We cannot at this time provide reliable estimates for the expected storm strengths a day or more in advance.

One of our goals was to provide quantified statements about the maturity of individual components of the GIC problem. Following this goal, we assigned in the sections above Applications Readiness Levels (ARLs) for pushing information between different links in the chain (Figure 2). It is our hope that the ARL concept 
will allow identification of key capability gaps and help guide the advancement of applied space weather sciences pertaining to GIC. We also envision that the assigned ARLs can be periodically revisited and updated, facilitating monitoring of the progress we are making in the field.

As a part of the NASA LWS Institute GIC Working Group activities, in addition to specifying the applications readiness of various components of the GIC problem, we also identified the key open scientific questions pertaining to the problem. Appendix A details the questions, most of which were already discussed above. The team also developed target "project templates" that can be used to address the identified questions, and those templates are provided in Appendix A. The LWS Institute team members used the project templates to initiate new collaborative research activities, some of which are reported in papers that accompany this overview paper. We hope that the material in Appendix A will facilitate further discussions on the challenges we face in the GIC systems science and help guide future work on the topic.

\section{Appendix A}

This paper is based on the findings of the very first Living With a Star (LWS) Institute Working Group. The new LWS Institutes program element was launched in 2014. The LWS Institute concept is built around small working group style meetings that focus on well-defined problems that demand intense, direct interactions between colleagues in neighboring disciplines to facilitate the development of a deeper understanding of the variety of processes that link the solar activity to Earth's environment. The LWS Institute working groups

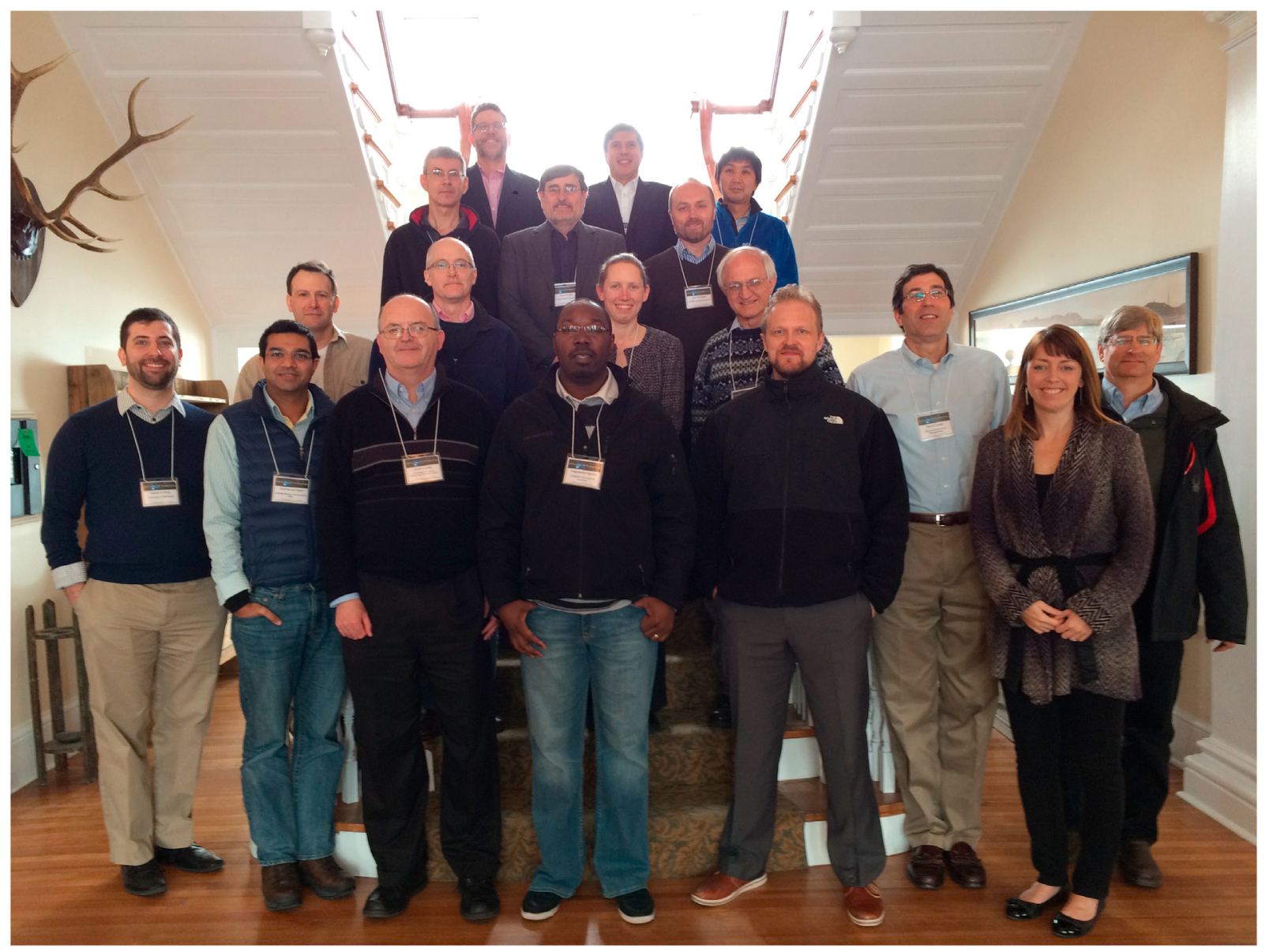

Figure A1. NASA LWS Institute GIC Working Group at the Stanley Hotel, Estes Park, Colorado, USA, 3 March 2015. Front row from left to right: D. Welling, N. Savani, G. Crowley, C. Ngwira, A. Pulkkinen, and K. Greb (UCAR). Second row from left to right: R. Weigel, B. Anderson, J. Simpson, P. Cilliers, D. Fugate, and C. Balch. Third row from left to right: A. Viljanen, A. Schultz, and A. Thomson. Back row from left to right: R. Leamon (NASA Headquarters), E. Bernabeu, R. and Kataoka. 
support the general LWS program goal to "Develop the scientific understanding necessary to enable the U.S. to effectively address those aspects of the connected Sun Earth system that directly affects life and society."

The LWS Institute Geomagnetically Induced Currents (GIC) Working Group (WG) was selected competitively as the pilot activity for the new LWS element. The GIC WG was tasked to (1) identify, advance, and address the open scientific and engineering questions pertaining to $\mathrm{GIC},(2)$ advance predictive modeling of GIC, and (3) advocate and act as a catalyst to identify resources for addressing the multidisciplinary topic of GIC. The group had two 1 week long in-person workshops 2015 in Colorado (Figure A1) and several videoconferences to develop the group materials that identify the status of the field and key challenges for improving scientific, engineering understanding on the topic. In addition to developing the overview of the status of the field, GIC WG identified key open scientific questions pertaining to the GIC problem. In this section those key questions, some of which were discussed also in the main sections of the paper, are identified. GIC WG also developed brief higher level "project templates" that can be used to address the identified questions. The templates are described in this section as well.

\section{A.1. Key Open Science Questions Pertaining to GIC}

The 1-D, 2-D, 3-D modeling of the geomagnetic induction and GIC:

1. How much does our GIC modeling improve by moving from the commonly used 1-D to 2-D and 3-D modeling of the geomagnetic induction?

2. Under what geological and geospace conditions does 1-D approach to geoelectric field and GIC modeling become insufficient?

How can we improve the work on extreme GIC event scenarios?

1. How can we improve geoelectric field and GIC statistics and reduce associated uncertaintities?

2. How can we improve modeling and understanding of the physics of extremes?

3. What are the theoretical upper bounds for the extreme geoelectric field and GIC events?

What is the optimal number and distribution of ground magnetic field measurements for GIC modeling purposes?

1. How can we carry out meaningful cost versus benefit analysis in terms of distribution of ground magnetic field measurement locations versus improvement in GIC modeling?

2. What is the minimum required quality of the measurements? Are observatory quality measurements in terms of stability, resolution, etc., needed or can we use lower cost partially off-the-shelf solutions?

GIC index development:

1. What could be new and improved (over $K p, \mathrm{~d} B / \mathrm{d} t$, and others that have used traditionally) indicators for GIC activity? Optimally, indicators need to be able to convey actionable information to the end users.

2. How can we package ground magnetic field information into data products that are useful for the end users?

Model validation:

1. How can we study and characterize key model (interplanetary transient, geospace response, and geomagnetic induction) accuracy and performance for GIC applications?

2. How can we build realistic error bars for our end products?

How to improve predictive GIC modeling?

1. How can we improve lead time and accuracy of our geoelectric field and GIC predictions?

2. Would focusing on specific interplanetary transient features (such as shock, sheath, and/or magnetic cloud) and geospace processes (such as sudden impulse, pulsations, and/or substorms) allow any simplification to the forecasting challenge?

\section{A.2. Project Templates}

The following projects were designed to address some of the open science questions identified above. The project templates should be understood only as brief higher level ideas on how the identified questions could be addressed. Also other approaches are entirely possible and we do not claim the approaches described below are necessarily the best or the most meaningful ones. 


\section{A.2.1. The 1-D, 2-D, 3-D Modeling of the Geomagnetic Induction and GIC}

In the frequency domain, GIC may be estimated using the measured geoelectric field $E$ on Earth's surface along with power system-dependent coefficients $a$ and $b$.

$$
\mathrm{GIC}(f)=a E x(f)+b E y(f)
$$

Historically, the quantity that is most often measured is the geomagnetic field $B$ on Earth's surface. The geoelectric field $E$ and GIC are much less often measured, and so an estimation is needed. Given measurements of $B$, one can estimate the geoelectric field using a transfer function $Z$, which has four components:

$$
\begin{aligned}
& E x(f)=Z x x(f) B x(f)+Z x y(f) B y(f) \\
& E y(f)=Z y x(f) B x(f)+Z y y(f) B y(f)
\end{aligned}
$$

Estimations of the transfer function (TF) require a magnetotelluric survey, in which the geoelectric and geomagnetic field are measured at a site over approximately 1 month. Statistical methods are then used to estimate the transfer function. This transfer function can capture fluctuations in the geoelectric field that can only be explained by three-dimensional variations in the ground conductivity.

Past estimates of the geoelectric field and GICs have generally relied upon so-called 1-D conductivity models, which specify the conductivity as a function of depth. From these models, it is straightforward to compute the transfer function $Z n(f)$ and then make estimates of $E$ given $B$ :

$$
\begin{gathered}
E x(f)=Z n(f) B y(f) \\
E y(f)=-Z n(f) B x(f)
\end{gathered}
$$

The transfer functions used to estimate geoelectric fields in the United States in the past have been based on those published by Fernberg [2012]. These conductivity models have well-known limitations and were presented as a first-order approximation that could be used when alternative information is lacking. Unfortunately, regional maps of storm time geoelectric variation are often needed either where magnetotelluric surveys have not been made or where the survey sites are sparsely distributed. In some cases, estimates of Earth conductivity have been based on simplistic assumptions about stratigraphy, tectonic structure, and rock properties, and so corresponding synthetic impedance tensors are of unknown accuracy. In particular, one-dimensional parameter depth-dependent models of conductivity have been used to estimate storm time geoelectric fields at specific sites and within defined geographic regions. Only very recently have storm time estimates been made using three-dimensional conductivity models.

Since 2006, the EarthScope USArray program of the National Science Foundation has supported magnetotelluric surveys over large geographic parts of the United States [Schultz, 2010]. These surveys were accomplished by temporary 3 week deployments of electromagnetic measurement systems at discrete locations having nominal $70 \mathrm{~km}$ spacing. At each site, $1 \mathrm{~Hz}$ geomagnetic vector data were collected using a fluxgate magnetometer; simultaneously, $1 \mathrm{~Hz}$ horizontal-component geoelectric vector data were collected using two orthogonal pairs of electrodes planted into the ground. Data from the measurement sites have been used to estimate empirical magnetotelluric impedance tensors or transfer functions. These tensors, in turn, have been used to estimate Earth conductivity models for the United States [e.g., Yang et al., 2015; Bedrosian, 2016].

The specific project templates considered here are (1) generate revised estimates for earlier extreme scenarios using full surface impedance tensors and (2) when using the EarthScope 3-D ground models to estimate the geoelectric field at a given location determine how the quality of prediction depends on (2a) method used to derive the TF, (2b) how the geoelectric field data were prefiltered before computing TF, (2c) the geomagnetic activity level in the time interval used for estimating TF, and (2d) dimensionality of TF: 1-D, 2-D, and 3-D.

\section{A.2.2. GIC Indicator Development}

A GIC indicator, or index, is intended to serve as a convenient summary measure of more complex phenomena to indicate various discrete levels of intensity over an interval of time. Traditional magnetic field indices such as the 3-hourly $K p$ index or hourly $D s t$ index have certain limitations in their ability to characterize the link between storm intensity and power system impacts. The proposed measures are more closely related 
to the induction hazard and can prove to be useful when making comparisons with related phenomena. They can also facilitate forecasts in situations where magnitude and timing uncertainties limit predictive capability to a range of values within an interval of time and where regional conductivity is not well known. Although various geomagnetic indices such as the hourly peak value of $\mathrm{GIC}$, hourly range $\mathrm{GIC}$, geomagnetic hourly peak value, geomagnetic hourly range indicator, geomagnetic hourly standard deviation, and hourly standard deviation of GIC have been utilized in this capacity, only the $\mathrm{GIC}_{x}$ and $\mathrm{GIC}_{y}$ indices developed by Marshall et al. [2011] have been designed specifically to characterize the level of impact of geomagnetic induction in technological systems such as power grids. The development of a GIC indicator is thus necessary to advance the state of space weather specification for this application. The goal of this project template is to explore a variety of hypothesized summary measures from the geomagnetic field observations and compare these to calculated geoelectric field measures and power system impacts over various time intervals. A consideration of the types of impacts on electrical power systems has focused the search for such indicators to address two types of effects having direct link to physical impact on performance of the grid: one to indicate the maximum level of geoelectric fields and another to indicate intervals of sustained, elevated geoelectric fields. The development activities will consider geomagnetic data from a variety of geomagnetic latitudes and surface impedance data from a representative range of locations to ensure that such measures can be used to specify activity for particular locations.

\section{A.2.3. Extreme GIC Events}

Rare but extremely intense magnetic storms not only challenge our understanding of space weather but also can have a detrimental impact on power transmission systems. Extreme storms events can be studied in two different approaches. Under a deterministic approach, the relevant natural and engineering processes are conceived as evolving continuously in time and over space according to differential equations describing the first-principle dynamics of the system. The deterministic approach is especially relevant for predicting the characteristics of a specific magnetic storm at Earth or for conducing scenario simulations of a plausible hypothetical event. A chain of physical quantities is measured using a combination of ground- and spacebased observation systems, and computers are used for forward calculation of the known physical principles, encompassing the domains of the Sun, the solar wind, the magnetospheric-ionospheric system, the solid Earth, and the power grid. In contrast, under a statistical approach, extreme events that are recorded in historical heliophysical and geophysical data are treated as discrete occurrences in time. Interest in forecasting the future occurrence of extreme events, the once-in-100 year event, for example, generally requires extrapolation of a statistical model that has been fitted to data covering a duration of time that is less than a 100 years. For this reason, it is important that the statistical models be physically motivated.

Specific project templates include (1) global simulation of the theoretically "most extreme" magnetic storms, (2) study of ionospheric current maps from historical events, (3) simulation of ionospheric currents during specific historical magnetic storms, (4) analysis of historical magnetograms (analogue and digital) recording sudden impulses, (5) analysis of digital magnetometer data recording $\mathrm{d} B / \mathrm{d} t$, including events that contain extremely large local or regional enhancements that suggest correspondingly large geoelectric fields and hence GIC, (6) analysis of the latitude dependence of the statistics of extreme events, (7) mapping of regional geoelectric induction in simplified lithospheric impedance models using recorded storm time geomagnetic activity, (8) mapping of regional geoelectric induction for simplified geomagnetic activity using directly measured lithospheric impedance, and (9) analysis of multiparameter solar wind conditions for extreme event magnetospheric coupling function.

Completion of these projects would provide useful information of assessment of ground-level induction hazards. However, substantial improvement can be made with (1) improvements in numerical and statistical analysis methods, (2) improved exchange of data, especially those recording the effects of magnetic storm induction on electric power grid operation, (3) improved monitoring of the Sun, solar wind, and magnetospheric-ionospheric systems, (4) improved monitoring of ground-level geomagnetic activity, (5) routine monitoring of the geoelectric field at key locations, and (6) completion of national-scale magnetotelluric surveys. Each of these improvements needs to be quantified in terms of their impact on predicting specific space weather event and forecasting the effects of extreme space weather events. It is worth recognizing that some of these needed improvements would also be useful in other geophysical domains. So, for example, improved geomagnetic monitoring would benefit induction hazard science and, also, fundamental scientific research of both the Earth's surrounding space environment and solid Earth geophysics; 
completing the national magnetotelluric survey would benefit both induction hazard science and solid Earth geophysics.

\section{A.2.4. Early Forecasting Methodologies for CMEs}

GIC are driven by a source 150 million of kilometers away - the Sun. Understanding the entire chain of events from the Sun to mud is complex and requires studies that span spatial scales of over 8 orders of magnitude from an astronomical unit to meters. Current forecast methodologies ultimately rely on interplanetary measurements of space at the L1 point upstream of the Earth's magnetosphere as their source data (giving a maximum $\sim 1 \mathrm{~h}$ forecast). These methodologies usually use combinations of empirical relationships and coupled simulations to nowcast and forecast GIC. However, there is a great need to develop predictions with more than $24 \mathrm{~h}$ lead time. Therefore, we describe practical and relevant technological solutions of how to use solar and interplanetary data to assist with improving the long lead time predictions of GICs. We describe how arrival time prediction of shocks driven by coronal mass ejections is of significant importance to the GIC issue and especially the plasma characteristics just behind the shock. In this work, we specifically focus on describing the first steps of how to create proxy-L1 data from solar imagery, which can then be used as alternative inputs into near space GIC prediction methodologies or various GIC proxy indicators. We will generate the proxy-L1 data for the St. Patrick's day event in March 2015, which are then used to drive the SWMF simulation and a 1-D response function. Therefore, this project will estimate the $\mathrm{d} B / \mathrm{d} t$, at select ground stations relevant for GIC activity, by using only solar observations measured 2 days prior to the measured Earth response.

\section{A.2.5. Performance Analysis of Geospace Models}

Models of the geospace environment show great promise as operational tools for monitoring and predicting near-Earth currents that drive GIC. Both physics simulations and empirical models can provide global electromagnetic conditions given parameters for the upstream solar wind. Solar wind inputs can come either from observations at L1, providing 3000 s predictions, or from models of the Sun and heliosphere environment to provide predictions a day or more in advance. The models also allow assessment of the effects of extreme events, for which observations are limited or nonexistent.

There are two problems that must be addressed to apply these models to estimate GIC: (i) quantifying model accuracies for storms with extensive data coverage and (ii) estimating the range of solar wind forcing for which the model results are valid. The most thorough validation exercise to date is Pulkkinen et al. [2013], which presented rigorous comparisons of model predictions and observed ground magnetic field signals. The importance of system nonlinearities, feedback, and saturation effects on extrapolation to conditions beyond the range of extant observations is not yet known. This motivates validation analyses focusing on the most intense events for which data are available.

To this end, in this project template, the analyses of Pulkkinen et al. [2013] will be extended to assess the model/simulation results versus space weather intensity and by comparing model/simulation results to global measures of ionospheric electrodynamics as derived both with the Assimilative Mapping of lonospheric Electrodynamics (AMIE) model and the Active Magnetosphere and Polar Electrodynamics Response Experiment (AMPERE). The range of known validity is assessed by examining both the limits inherent in the models' assumptions and the limits over which they have been tested in past validation studies. The data model error from the Pulkkinen et al. [2013] study is binned by geomagnetic storm intensity (e.g., the Dst index) to assess model performance as a function of activity. In addition, the comparisons of modeled and observed ionospheric electrodynamics allow unprecedented assessment of the global-scale reliability of the modeled system dynamics as functions of local time and latitude. The results of these analyses quantify the level of validity of the present generation of operational-ready models and identify key areas in which the models need to be improved.

\section{References}

Anderson, B., K. Takahashi, and B. Toth (2000), Sensing global Birkeland currents with iridium ${ }^{\oplus}$ engineering magnetometer data, Geophys. Res. Lett., 27, 4045-4048, doi:10.1029/2000GL000094.

Bedrosian, P. A. (2016), Making it and breaking it in the Midwest: Continental assembly and rifting from modeling of EarthScope magnetotelluric data, Precambrian Res., 278, 378-361, doi:10.1016/j.precamres.2016.03.009.

Bedrosian, P. A., and J. J. Love (2015), Mapping geoelectric fields during magnetic storms: Synthetic analysis of empirical United States impedances, Geophys. Res. Lett., 42, 10,160-10,170, doi:10.1002/2015GL066636.

Beggan, C. (2015), Sensitivity of geomagnetically induced currents to varying auroral electrojet and conductivity models, Earth Planets Space, 67, 24, doi:10.1186/s40623-014-0168-9. 
Bernabeu, E., et al. (2015), Harmonic load flow during geomagnetic disturbances, vol. 3, CIGRE Sci. \& Eng.

Bolduc, L. (2002), GIC observations and studies in the Hydro-Quebec power system, J. Atmos. Sol. Terr. Phys., 64, 1793.

Bolduc, L., P. Langlois, D. Boteler, and R. Pirjola (2000), A study of geoelectro- magnetic disturbances in Quebec, 2. Detailed analysis of a large event, IEEE Trans. Power Delivery, 15, 272.

Borovsky, J. E., and M. H. Denton (2006), Differences between CME-driven storms and CIR-driven storms, J. Geophys. Res., 111, A07S08, doi:10.1029/2005JA011447.

Boteler, D., (1997), Distributed source transmission line theory for electromagnetic induction studies, in Supplement of the Proceedings of the 12th International Zurich Symposium and Technical Exhibition on Electromagnetic Compatibility, pp. 401-408.

Boteler, D., and E. Bradley (2016), On the interaction of power transformers and geomagnetically induced currents, IEEE Trans. Power Delivery, 1(5), 2188-2195.

Boteler, D., and R. Pirjola (2017), Modelling geomagnetically induced currents, Space Weather, 15, 258-276, doi:10.1002/2016SW001499. Boteler, D. H. (2001), Assessment of geomagnetic hazard to power systems in Canada, Nat. Hazards, 23, 101-120.

Boteler, D. H., and R. J. Pirjola (2014), Comparison of methods for modelling geomagnetically induced currents, Ann. Geophys., 32, 1177-1187, doi:10.5194/angeo-32-1177-2014.

Boteler, D. H., R. J. Pirjola, and H. Nevanlinna (1998), The effects of geomagnetic disturbances on electrical systems at the Earth's surface, $A d v$. Space Res., 22, 17.

Cabinet Office (2015), National Risk Register of Civil Emergencies. [Available at https://www.gov.uk/government/uploads/system/uploads/ attachment_data/file/419549/20150331_2015-NRR-WA_Final.pdf.]

Cagniard, L. (1953), Basic theory of the magneto-telluric method of geophysical prospecting, Geophysics, 18(3), 605.

Cardona, O. D., M. K. van Aalst, J. Birkmann, M. Fordham, G. McGregor, R. Perez, R. S. Pulwarty, E. L. F. Schipper, and B. T. Sinh (2012), Determinants of risk: Exposure and vulnerability, in Managing the Risks of Extreme Events and Disasters to Advance Climate Change Adaptation, A Special Report of Working Groups I and II of the Intergovernmental Panel on Climate Change (IPCC), edited by C. B. Field, pp. 65-108, Cambridge Univ. Press, Cambridge, U. K., and New York.

Carter, B. A., E. Yizengaw, R. Pradipta, A. J. Halford, R. Norman, and K. Zhang (2015), Interplanetary shocks and the resulting geomagnetically induced currents at the equator, Geophys. Res. Lett., 42, 6554-6559, doi:10.1002/2015GL065060.

Electric Power Research Institute (2011), Sunburst Network for Geomagnetic Currents, EPRI Product Abstract, Product ID 1023278. [Available at http://www.epri.com/abstracts/Pages/ProductAbstract.aspx?Productld=000000000001023278.]

Federal Energy Regulatory Commission (2015), Reliability standard for transmission system planned performance for geomagnetic disturbance events, 18 CFR Part 40, Docket No. RM15-11-000.

Fernberg, P. (2012), One-dimensional Earth resistivity models for selected areas of continental United States and Alaska, pp. 1-190, EPR Technical Update 1026430, Palo Alto, Calif.

Forbes, K. F., and O. C. St. Cyr (2008), Solar activity and economic fundamentals: Evidence from 12 geographically disparate power grids, Space Weather, 6, S10003, doi:10.1029/2007SW000350.

Forbes, K. F., and O. C. St. Cyr (2010), An anatomy of space weather's electricity market impact: Case of the PJM power grid and the performance of its $500 \mathrm{kV}$ transformers, Space Weather, 8, S09004, doi:10.1029/2009SW000498.

Ganushkina, N. Y., et al. (2015), Defining and resolving current systems in geospace, Ann. Geophys., 33, 1369-1402, doi:10.5194/angeo-331369-2015.

Gaunt, C. T. (2014), Reducing uncertainty-responses for electricity utilities to severe solar storms, J. Weather Space Clim., 4, A01.

Gaunt, C. T., and G. Coetzee (2007), Transformer failures in regions incorrectly considered to have low GIC-risk, in Power Tech, 2007 IEEE Lausanne: Proceedings, pp. 807-812, Inst. of Elec. and Elec. Eng., Piscataway, N. J.

Glocer, A., et al. (2016), Community-wide validation of geospace model local $K$-index predictions to support model transition to operations, Space Weather, 14, 469-480, doi:10.1002/2016SW001387.

Huttunen, K. E. J., S. P. Kilpua, A. Pulkkinen, A. Viljanen, and E. Tanskanen (2008), Solar wind drivers of large geomagnetically induced currents during the solar cycle 23, Space Weather, 6, S10002, doi:10.1029/2007SW000374.

Kappenman, J. G. (2003), Storm sudden commencement events and the associated geomagnetically induced current risks to ground-based systems at low-latitude and midlatitude locations, Space Weather, 1(3), 1016, doi:10.1029/2003SW000009.

Kataoka, R., and C. Ngwira (2016), Extreme geomagnetically induced currents, Prog. Earth Planet. Sci., 3, 23, doi:10.1186/s40645016-0101.

Kataoka, R., and A. Pulkkinen (2008), Geomagnetically induced currents during intense storms driven by coronal mass ejections and corotating interacting regions, J. Geophys. Res., 113, A03S12, doi:10.1029/2007JA012487.

Kelbert, A., C. Balch, A. A. Pulkkinen, G. D. Egbert, J. J. Love, E. J. Rigler and I. Fujii (2017), Methodology for time-domain estimation of stormtime geoelectric fields using the 3D magnetotelluric response tensors, Space Weather, 15, doi:10.1002/2017SW001594

Knipp, D. (2015), Forward to space weather collection on geomagnetically induced currents: Commentary and research, Space Weather, 13, 742-746, doi:10.1002/2015SW001318.

Lehtinen, M., and R. Pirjola (1985), Currents produced in earthed conductor networks by geomagnetically-induced electric fields, Ann Geophys., 3(4), 479.

Lionello, R., C. Downs, J. A. Linker, T. Torok, P. Riley, and Z. Mikic (2013), Magnetohydrodynamic simulations of interplanetary coronal mass ejections, Astrophys. J., 777(76), 11, doi:10.1088/0004-637X/777/1/76.

Liu, C. M., L. G. Liu, and R. Pirjola (2009), Geomagnetically induced currents in the high-voltage power grid in China, IEEE Trans. Power Delivery, $24,4$.

Liu, Y., et al. (2014), Observations of an extreme storm in interplanetary space caused by successive coronal mass ejections, Nat. Commun., 5 , 3481, doi:10.1038/ncomms4481.

Love, J. J., and C. A. Finn (2011), The USGS geomagnetism program and its role in space weather monitoring, Space Weather, 9, S07001, doi:10.1029/2011SW000684.

Love, J. J., E. J. Rigler, A. Pulkkinen, and C. C. Balch (2014), Magnetic storms and induction hazards, Eos Trans. AGU, 95(48), 445-446, doi:10.1002/2014EO480001.

Love, J. J., P. Coisson, and A. Pulkkinen (2016a), Global statistical maps of extreme-event magnetic observatory 1-min first differences in horizontal intensity, Geophys. Res. Lett., 43, 4126-4135, doi:10.1002/2016GL068664.

Love, J. J., et al. (2016b), Geoelectric hazard maps for the continental United States, Geophys. Res. Lett., 43, 9415-9424, doi:10.1002/ 2016GL070469.

Marshall, R. A., E. A. Smith, M. J. Francis, C. L. Waters, and M. D. Sciffer (2011), A preliminary risk assessment of the Australian region power network to space weather, Space Weather, 9, S10004, doi:10.1029/2011SW000685. 
Marti, L., A. Rezaei-Zare, and A. Narang (2013), Simulation of transformer hotspot heating due to geomagnetically induced currents, IEEE Trans. Power Delivery, 28(1), 320-327.

Marti, L., C. Yiu, A. Rezaei-Zare, and D. Boteler (2014), Simulation of geomagnetically induced currents with piecewise layered-earth models, IEEE Trans. Power Delivery, 29(4), 1886-1893.

McKay, A. J. (2004), Geoelectric fields and geomagnetically induced currents in the United Kingdom, PhD thesis, 237 pp., Univ. of Edinburgh. [Available at http://www.era.lib.ed.ac.uk/1842/639.]

Meier, A. V. (2006), Electric Power Systems: A Conceptual Introduction, John Wiley, Hoboken, N. J.

Millward, G., D. Biesecker, V. Pizzo, and C. A. de Koning (2013), An operational software tool for the analysis of coronagraph images: Determining CME parameters for input into the WSA-Enlil heliospheric model, Space Weather, 11, 57-68, doi:10.1002/swe.20024. Molinski, T. (2002), Why utilities respect geomagnetically induced currents, J. Atmos. Sol. Terr. Phys., 64, 1765-1778.

National Research Council (2008), Severe Space Weather Events: Understanding Societal and Economic Impacts-A Workshop Report, Natl. Acad. Press, Washington, D. C.

National Science and Technology Council (2015a), National Space Weather Strategy, Executive Office of the President (EOP), USA. [Available at https://www. whitehouse.gov/sites/default/files/microsites/ostp/final_nationalspaceweatherstrategy_20151028.pdf.]

National Science and Technology Council (2015b), National Space Weather Action Plan, Executive Office of the President (EOP), USA. [Available at https://www.whitehouse.gov/sites/default/files/microsites/ostp/final_nationalspaceweatheractionplan_20151028.pdf.]

Ngwira, C., A. Pulkkinen, M. Kuznetsova, and A. Glocer (2014), Modeling extreme "Carrington-type" space weather events using threedimensional global MHD simulations, J. Geophys. Res. Space Physics, 119, 4456-4474, doi:10.1002/2013JA019661.

Ngwira, C., A. Pulkkinen, E. Bernabeu, J. Eichner, A. Viljanen, and G. Crowley (2015), Characteristics of extreme geoelectric fields and their possible causes: Localized peak enhancements, Geophys. Res. Lett., 42, 6916-6921, doi:10.1002/2015GL065061.

Ngwira C. M., A. Pulkkinen, L.-A. McKinnell, and P. J. Cilliers (2008), Improved modelling of geomagnetically induced currents in the South African power network, Space Weather, 6, S11004, doi:10.1029/2008SW000408.

Nikitina, L., L. Trichtchenko, and D. H. Boteler (2016), Assessment of extreme values in geomagnetic and geoelectric field variations for Canada, Space Weather, 14, 481-494, doi:10.1002/2016SW001386.

North American Electric Reliability Corporation (2012), 2012 Special Reliability Assessment Interim Report: Effects of geomagnetic disturbances on the bulk power system, February 2012

North American Electric Reliability Corporation (2013), White Paper Supporting Network Applicability of EOP-010-1, 2013. [PDF available at http://www.nerc.com/pa/Stand/Pages/Project-2013-03-Geomagnetic-Disturbance-Mitigation.aspx.]

North American Electric Reliability Corporation (2016a), Benchmark Geomagnetic Disturbance Event Description, Project 2013-03 GMD Mitigation Standards Drafting Team, May 2016. [PDF available at http://www.nerc.com/pa/Stand/Pages/Project-2013-03-GeomagneticDisturbance-Mitigation.aspx.]

North American Electric Reliability Corporation (2016b), Transformer Thermal Impact Assessment white paper, Project 2013-03 GMD Mitigation Standards Drafting Team, May 2016. [PDF available at http://www.nerc.com/pa/Stand/Pages/Project-2013-03-GeomagneticDisturbance-Mitigation.aspx.]

Oyedokun, D. (2015), Geomagnetically induced currents (GICs) in large power systems including transformer time response, PhD Thesis, Univ. of Cape Town.

Pirjola, R. (2013), Practical model applicable to investigating the coast effect on the geoelectric field in connection with studies of geomagnetically induced currents, Adv. Appl. Phys., 1(1), 9-28.

Pizzo, V., G. Millward, A. Parsons, D. Biesecker, S. Hill, and D. Odstrcil (2011), Wang-Sheeley-Arge-Enlil cone model transitions to operations, Space Weather, 9, S03004, doi:10.1029/2011SW000663.

Pulkkinen, A., S. Lindahl, A. Viljanen, and R. Pirjola (2005), Geomagnetic storm of 29-31 October 2003: Geomagnetically induced currents and their relation to problems in the Swedish high-voltage power transmission system, Space Weather, 3, S08C03, doi:10.1029/2004SW000123.

Pulkkinen, A., A. Taktakishvili, D. Odstrcil and W. Jacobs (2009a), Novel approach to geomagnetically induced current forecasts based on remote solar observations, Space Weather, 7, S08005, doi:10.1029/2008SW000447.

Pulkkinen, A., M. Hesse, S. Habib, L. Van der Zel, B. Damsky, F. Policelli, D. Fugate, and W. Jacobs (2009b), Solar Shield: Forecasting and mitigating space weather effects on high-voltage power transmission systems, Nat. Hazards, doi:10.1007/s11069-009-9432-x.

Pulkkinen, A., R. Kataoka, S. Watari, and M. Ichiki (2010), Modeling geomagnetically induced currents in Hokkaido, Japan, Adv. Space Res., 46, 1087-1093.

Pulkkinen, A., E. Bernabeu, J. Eichner, C. Beggan, and A. Thomson (2012), Generation of 100-year geomagnetically induced current scenarios, Space Weather, 10, S04003, doi:10.1029/2011SW000750.

Pulkkinen, A., et al. (2013), Community-wide validation of geospace model ground magnetic field perturbation predictions to support model transition to operations, Space Weather, 11, 369-385, doi:10.1002/swe.20056.

Pulkkinen, A., E. Bernabeu, J. Eichner, A. Viljanen, and C. M. Ngwira (2015), Regional-scale high-latitude extreme geoelectric fields pertaining to geomagnetically induced currents, Earth Planets Space, doi:10.1186/s40623-015-0255-6.

Püthe, C., C. Manoj, and A. Kuvshinov (2014), Reproducing electric field observations during magnetic storms by means of rigorous 3-D modelling and distortion matrix co-estimation, Earth Planets Space, 66, 162, doi:10.1186/s40623-014-0162-2.

Rastätter, L., M. Kuznetsova, G. Toth, and A. Pulkkinen (2014), CalcDeltaB: An efficient postprocessing tool to calculate ground-level magnetic perturbations from global magnetosphere simulations, Space Weather, 12, 553-565, doi:10.1002/2014SW001083.

Richmond, A. D. (1992), Assimilative mapping of ionospheric electrodynamics, Adv. Space Res., 12(6), (6)69-(6)68.

Richmond, A. D., G. Lu, B. A. Emery, and D. J. Knipp (1998), The AMIE procedure: Prospects for space weather specification and prediction, Adv. Space Res., 22(1), 103-112.

Royal Academy of Engineering (2013), Extreme space weather: Impacts on engineered systems and infrastructure, report published by Royal Academy of Engineering, isbn:1-903496-95-0.

Samimi, A., and J. Simpson (2016), Parallelization of 3-D Global FDTD Earth-ionosphere waveguide models at resolutions on the order of $\sim 1 \mathrm{~km}$ and higher, IEEE Anten. Wireless Propag. Lett., 15, 1959-1962, doi:10.1109/LAWP.2016.2545526.

Savani, N. P., A. P. Rouillard, J. A. Davies, M. J. Owens, R. J. Forsyth, C. J. Davis, and R. A. Harrison (2009), The radial width of a coronal mass ejection between 0.1 and $0.4 \mathrm{AU}$ estimated from the Heliospheric Imager on STEREO, Ann. Geophys., 27(11), 4349-4358, doi:10.5194/ angeo-27-4349-2009.

Savani, N. P., M. J. Owens, A. P. Rouillard, R. J. Forsyth, and J. A. Davies (2010), Observational evidence of a coronal mass ejection distortion directly attributable to a structured solar wind, Ap. J. Lett., 714(1), L128-L132, doi:10.1088/2041-8205/714/1/L128.

Savani, N. P., A. Vourlidas, A. Pulkkinen, T. Nieves-Chinchilla, B. Lavraud, and M. J. Owens (2013), Tracking the momentum flux of a CME and quantifying its influence on geomagnetically induced currents at Earth, Space Weather, 11, 245-261, doi:10.1002/swe.20038. 
Savani, N. P., A. Vourlidas, A. Szabo, M. L. Mays, I. G. Richardson, B. J. Thompson, A. Pulkkinen, R. Evans, and T. Nieves-Chinchilla (2015), Predicting the magnetic vectors within coronal mass ejections arriving at Earth: 1. Initial architecture, Space Weather, 13, 374-385, doi:10.1002/2015SW001171.

Schrijver, C., and S. Mitchell (2013), Disturbances in the US electric grid associated with geomagnetic activity, J. Space Weather Space Clim., 3, A19, doi:10.1051/swsc/2013041.

Schrijver, C. J., et al. (2015), Understanding space weather to shield society: A global road map for 2015-2025 commissioned by COSPAR and ILWS, Adv. Space Res., 55, 2745-2807.

Schultz, A. (2010), EMScope: a continental scale magnetotelluric observatory and data discovery resource, Data Sci. J., 8, IGY6-IGY20.

Shiota, D., and R. Kataoka (2016), Magnetohydrodynamic simulation of interplanetary propagation of multiple coronal mass ejections with internal magnetic flux rope (SUSANOO-CME), Space Weather, 14, 56-75, doi:10.1002/2015SW001308.

Simpson, J. J. (2011), On the possibility of high-level transient coronal mass ejection-induced ionospheric current coupling to electric power grids, J. Geophys. Res., 116, A11308, doi:10.1029/2011JA016830.

Thernisien, A., A. Vourlidas, and R. A. Howard (2009), Forward modeling of coronal mass ejections using STEREO/SECCHI data, Sol. Phys., 256(1-2), 111, doi:10.1007/s11207-009-9346-5.

Thomson, A. W. P., C. T. Gaunt, P. Cilliers, J. A. Wild, B. Opperman, L.-A. McKinnell, P. Kotze, C. M. Ngwira, and S. I. Lotz (2010), Present day challenges in understanding the geomagnetic hazard to national power grids, J. Adv. Space Res., doi:10.1016/j.asr.2009.11.023.

Thomson, A., S. Reay, and E. Dawson (2011), Quantifying extreme behavior in geomagnetic activity, Space Weather, 9, S10001, doi:10.1029/ 2011 SW000696.

Torta, J. M., L. Serrano, J. R. Regué, A. M. Sánchez, and E. Roldán (2012), Geomagnetically induced currents in a power grid of northeastern Spain, Space Weather, 10, S06002, doi:10.1029/2012SW000793.

Tóth, G., D. L. De Zeeuw, T. I. Gombosi, W. B. Manchester, A. J. Ridley, I. V. Sokolov, and I. I. Roussev (2007), Sun-to-thermosphere simulation of the 28-30 October 2003 storm with the Space Weather Modeling Framework, Space Weather, 5, S06003, doi:10.1029/2006SW000272.

Trichtchenko, L., and D. H. Boteler (2004), Modeling geomagnetically induced currents using geomagnetic indices and data, IEEE Trans. Plasma Sci., 32(4), 1459-1467.

United States of America Federal Energy Regulatory Commission (2013), Reliability standards for geomagnetic disturbances, Order 779,16 May. [Available at https://www.ferc.gov/whats-new/comm-meet/2013/051613/E-5.pdf.]

University of Cambridge, Center for Risk Studies (2016), Helios solar storm scenario, Cambridge Centre for Risk Studies Publications

Viljanen, A., and R. Pirjola (1989), Statistics on geomagnetically-induced currents in the Finnish $400 \mathrm{kV}$ power system based on recordings of geomagnetic variations, J. Geomagn. Geoelectr., 41, 411-420.

Viljanen, A., A. Pulkkinen, R. Pirjola, K. Pajunpää, P. Posio, and A. Koistinen (2006), Recordings of geomagnetically induced currents and a now casting service of the Finnish natural gas pipeline system, Space Weather, 4, S10004, doi:10.1029/2006SW000234.

Vourlidas, A. (2015), Mission to the Sun-Earth L5 Lagrangian point: An optimal platform for space weather research, Space Weather, 13, 197-201, doi:10.1002/2015SW001173.

Wik, M., A. Viljanen, R. Pirjola, A. Pulkkinen, P. Wintoft, and H. Lundstedt (2008), Calculation of geomagnetically induced currents in the $400 \mathrm{kV}$ power grid in southern Sweden, Space Weather, 6, S07005, doi:10.1029/2007SW000343.

Williams, M. L., et al. (2010), Unlocking the secrets of the North American continent: An EarthScope science plan for 2010-2020, pp. 1-78, EarthScope.

Yang, B., G. D. Egbert, A. Kelbert, and N. M. Meqbel (2015), Three-dimensional electrical resistivity of the north-central USA from EarthScope long period magnetotelluric data, Earth Planet. Sci. Lett., 422, 87-93.

Zhang, J. J., C. Wang, and B. B. Tang (2012), Modeling geomagnetically induced electric field and currents by combining a global MHD model with a local one-dimensional method, Space Weather, 10, S05005, doi:10.1029/2012SW000772.

Zheng, Y., A. Pulkkinen, A. Taktakishvili, P. MacNeice, M. Hesse, and M. Kuznetsova (2013), Forecasting CMEs in an operational setting: What has been learned?, Space Weather, 11, 557-574, doi:10.1002/swe.20096 